ESAIM: COCV 20 (2014) 633-661

DOI: $10.1051 / \mathrm{cocv} / 2013078$
ESAIM: Control, Optimisation and Calculus of Variations

www.esaim-cocv.org

\title{
A DETERMINISTIC AFFINE-QUADRATIC OPTIMAL CONTROL PROBLEM*
}

\author{
YUANCHANG WANG ${ }^{1,2}$ AND JiONGMIN YONG ${ }^{2}$
}

\begin{abstract}
A deterministic affine-quadratic optimal control problem is considered. Due to the nature of the problem, optimal controls exist under some very mild conditions. Further, it is shown that under some assumptions, the optimal control is unique which leads to the differentiability of the value function. Therefore, the value function satisfies the corresponding Hamilton-Jacobi-Bellman equation in the classical sense, and the optimal control admits a state feedback representation. Under some additional conditions, it is shown that the value function is actually twice differentiable and the so-called quasiRiccati equation is derived, whose solution can be used to construct the state feedback representation for the optimal control.
\end{abstract}

Mathematics Subject Classification. 49J15, 49K15, 49L20, 49N10.

Received June 22, 2013. Revised October 11, 2013.

Published online May 21, 2014.

\section{INTRODUCTION}

Consider the following controlled ordinary differential equation (ODE, for short):

$$
\left\{\begin{array}{l}
\dot{X}(s)=A(s, X(s))+B(s, X(s)) u(s), \quad s \in[t, T], \\
X(t)=x
\end{array}\right.
$$

with cost functional

$$
J(t, x ; u(\cdot))=\int_{t}^{T}\left[Q(s, X(s))+\langle S(s, X(s)), u(s)\rangle+\frac{1}{2}\langle R(s, X(s)) u(s), u(s)\rangle\right] \mathrm{d} s+G(X(T)),
$$

where $A:[0, T] \times \mathbb{R}^{n} \rightarrow \mathbb{R}^{n}, B:[0, T] \times \mathbb{R}^{n} \rightarrow \mathbb{R}^{n \times m}, Q:[0, T] \times \mathbb{R}^{n} \rightarrow \mathbb{R}, S:[0, T] \times \mathbb{R}^{n} \rightarrow \mathbb{R}^{m}$, $R:[0, T] \times \mathbb{R}^{n} \rightarrow \mathbb{S}_{+}^{m}\left(\mathbb{S}^{m}\right.$ is the set of all symmetric matrices, and $\mathbb{S}_{+}^{m}$ is the set of all positive definite matrices), and $G:[0, T] \times \mathbb{R}^{n} \rightarrow \mathbb{R}$ are some given maps. Let $\mathcal{U}[t, T]$ be the set of all admissible controls (which will be

Keywords and phrases. Affine quadratic optimal control, dynamic programming, Hamilton-Jacobi-Bellman equation, quasi-Riccati equation, state feedback representation.

* The first author was supported in part by NSFC under Grant 71163046 and China State Scholarship Fund under Grant [2009] 5004, the second author was supported in part by NSF under Grant DMS-1007514.

1 School of Mathematics, Yunnan Normal University, Kunming, 650500, P.R. China.

2 Department of Mathematics, University of Central Florida, Orlando, FL 32816, USA. Jiongmin. Yong@ucf.edu 
specified in the next section) on $[t, T]$. Under some mild conditions, for any $(t, x) \in[0, T) \times \mathbb{R}^{n}$ and $u(\cdot) \in \mathcal{U}[t, T]$, the state equation (1.1) admits a unique solution $X(\cdot) \equiv X(\cdot ; t, x, u(\cdot))$ and the cost functional (1.2) is welldefined. Then we can pose the following optimal control problem.

Problem (AQ). For any given $(t, x) \in[0, T) \times \mathbb{R}^{n}$, find a $u^{*}(\cdot) \in \mathcal{U}[t, T]$ such that

$$
J\left(t, x ; u^{*}(\cdot)\right)=\inf _{u(\cdot) \in \mathcal{U}[t, T]} J(t, x ; u(\cdot)) \equiv V(t, x) .
$$

Any $u^{*}(\cdot)$ satisfying the above is called an optimal control for $(t, x)$, and the corresponding $X^{*}(\cdot) \equiv$ $X\left(\cdot ; t, x, u^{*}(\cdot)\right)$ is called an optimal trajectory for $(t, x)$. The pair $\left(X^{*}(\cdot), u^{*}(\cdot)\right)$ is called an optimal pair of Problem (AQ) for the initial pair $(t, x)$. The function $V(\cdot, \cdot)$ is called the value function of Problem (AQ).

We note that the right hand side of the state equation is affine with respect to the control and the integrand in the cost functional is up to quadratic with respect to the control. Therefore, we call such a problem an affine-quadratic optimal control problem (AQ problem, for short). We see that if

$$
\left\{\begin{array}{l}
A(t, x)=A(t) x, \quad B(t, x)=B(t), \quad Q(t, x)=\frac{1}{2}\langle Q(t) x, x\rangle, \\
S(t, x)=S(t) x, \quad R(t, x)=R(t), \quad G(x)=\frac{1}{2}\langle G x, x\rangle,
\end{array} \quad \forall(t, x) \in[0, T] \times \mathbb{R}^{n},\right.
$$

for some matrix-valued functions $A(\cdot), B(\cdot), Q(\cdot), S(\cdot), R(\cdot)$, and some matrix $G$, then our Problem (AQ) is reduced to a standard linear-quadratic optimal control problem (LQ problem, for short).

It is well-known that for LQ problem, under suitable conditions, one has the existence of a unique optimal control which admits a state feedback representation via the solution of a differential Riccati equation (see [14], and also [21]). On the other hand, for optimal control problem of general nonlinear ordinary differential equation with a Bolza type cost functional, one generally does not expect the existence of an optimal control; However, under some mild conditions, one can characterize the value function of the optimal control problem as the unique viscosity solution to the so-called Hamilton-Jacobi-Bellman (HJB, for short) equation ([4], see also $[5,16]$, and the references cited therein). Note that our Problem (AQ) is between general (nonlinear) optimal control problems and LQ problems. Therefore, one expects that our results should be "between" those for the above-mentioned two kinds of problems. A little more precisely, under some mild conditions, we will have the existence of optimal controls, and the optimality system (which is a two-point boundary value problem) from Pontryagin's minimum principle will admit a solution. By impose some proper conditions, we will show that the optimality system has a unique solution, which leads to the uniqueness of the optimal control. Then following from a result of [11], we obtain the differentiability of the value function. Hence, the value function satisfies the Hamilton-Jacobi-Bellman (HJB, for short) in the classical sense, and the optimal control will admit a state feedback representation. Furthermore, under some additional conditions, we show that the map $u(\cdot) \mapsto J(t, x ; u(\cdot))$ is uniformly convex, which yields the second order differentiability of the value function. Then, by differentiating the HJB equation, we obtain a quasi-Riccati equation, whose solution is exactly the one we need to represent the optimal control in a state feedback form. Our result covers those found in $[22,23]$ where Problem (AQ) with the state equation being linear and with the maps $x \mapsto Q(t, x)$ and $x \mapsto G(x)$ being convex, and $S(t, x) \equiv 0$ was studied. Also, we mention that without giving details, Problem (AQ) for stochastic differential equations was briefly discussed in [19].

We refer to $[4,11]$ for excellent surveys on the value function of (deterministic) optimal control theory. See also $[5,6,9,17]$ for some relevant results concerning the differentiability of value functions. We mention that the so-called state-dependent Riccati equation (see [1-3,10], and references cited therein) have been used to study Problem (AQ), with the main concerns being the approximation of optimal control and stabilization of the system. Final, we mention an excellent book [8] (and the references cited therein) for relevant general (coercive) optimization problems which are closely relevant to the current paper.

The rest of the paper is organized as follows. Section 2 collects some preliminary results. In Section 3, we present the existence of optimal controls for our Problem (AQ) and recall a Pontryagin type minimum 
principle. In Section 4, we study the differentiability of the value function via the uniqueness of the solution to the optimality system and the convexity of the cost functional. In Section 5, we calculate the first and the second order Fréchet derivatives of the cost functional with respective to the control. Based on these, the uniform positive definiteness of the Hessian $D_{u u} J(t, x ; u(\cdot))$ of the cost functional with respect to the control variable is obtained in Section 6, under certain sufficient conditions. In Section 7, we derive the so-called quasi-Riccati equation in a very natural way, via which a state feedback representation of the optimal control is obtained. A couple of illustrative examples are presented as well. Finally, some concluding remarks are collected in Section 8.

\section{Preliminaries}

Throughout this paper, we let $U \subseteq \mathbb{R}^{m}$ be a nonempty convex and closed set, not necessarily bounded and it could be $U=\mathbb{R}^{m}$. For convenience, we assume hereafter that $0 \in U$. Now, we introduce the following standing assumptions.

(H1) The maps $A:[0, T] \times \mathbb{R}^{n} \rightarrow \mathbb{R}^{n}$ and $B:[0, T] \times \mathbb{R}^{n} \rightarrow \mathbb{R}^{n \times m}$ are continuous. There exist constants $L_{A}, L_{B}, \widetilde{L}_{B}>0$ such that

$$
\begin{aligned}
& |A(t, x)-A(t, \bar{x})| \leq L_{A}|x-\bar{x}|, \quad \forall t \in[0, T], x, \bar{x} \in \mathbb{R}^{n}, \\
& |B(t, x)-B(t, \bar{x})| \leq \widetilde{L}_{B}|x-\bar{x}|, \quad \forall t \in[0, T], x, \bar{x} \in \mathbb{R}^{n},
\end{aligned}
$$

and

$$
\left\langle[B(t, x)-B(t, \bar{x})]^{T}(x-\bar{x}), u\right\rangle \leq L_{B}|x-\bar{x}|^{2}, \quad \forall(t, u) \in[0, T] \times U, x, \bar{x} \in \mathbb{R}^{n} .
$$

Note that condition (2.3) is equivalent to the following:

$$
\sup _{u \in U, x, \bar{x} \in \mathbb{R}^{n}, x \neq \bar{x}} \frac{\left\langle[B(t, x)-B(t, \bar{x})]^{T}(x-\bar{x}), u\right\rangle}{|x-\bar{x}|^{2}} \leq L_{B} .
$$

On the other hand, under $(2.2)$, the set

$$
\mathcal{X}=\left\{\frac{[B(t, x)-B(t, \bar{x})]^{T}(x-\bar{x})}{|x-\bar{x}|^{2}} \mid x, \bar{x} \in \mathbb{R}^{n}, x \neq \bar{x}\right\} \subseteq \mathcal{B}_{\tilde{L}_{B}}^{m}(0),
$$

where $\mathcal{B}_{r}^{m}(0)$ is the closed ball in $\mathbb{R}^{m}$ centered at 0 with radius $r$. Therefore, in the case $U$ is bounded, (2.4) is satisfied with

$$
L_{B} \geq \widetilde{L}_{B} \sup _{u \in U}|u|
$$

In the case $U=\mathbb{R}^{m},(2.4)$ is equivalent to the following:

$$
[B(t, x)-B(t, \bar{x})]^{T}(x-\bar{x})=0, \quad \forall t \in[0, T], x, \bar{x} \in \mathbb{R}^{n} .
$$

If we denote

$$
B(t, x)=\left(B^{1}(t, x), B^{2}(t, x), \cdots, B^{m}(t, x)\right), \quad B^{i}:[0, T] \times \mathbb{R}^{n} \rightarrow \mathbb{R}^{n}, 1 \leq i \leq m,
$$

then (2.6) is equivalent to the following:

$$
\left\langle B^{i}(t, x)-B^{i}(t, \bar{x}), x-\bar{x}\right\rangle=0, \quad 1 \leq i \leq m .
$$

This is the case if $B_{x}^{i}(t, x)$ is skew symmetric, for each $1 \leq i \leq m$. In particular, this is the case, of course, if $B(t, x)=B(t)$ is independent of $x$. Note that even if $B(t, x)=B(t)$ is independent of $x$, due to the fact that $x \mapsto A(t, x)$ is not necessarily linear, we still have a nonlinear state equation. Condition (2.3) plays an important role in Proposition 2.1 below.

Next, we introduce the following hypothesis for the functions appearing in the cost functional. 
(H2) Maps $Q:[0, T] \times \mathbb{R}^{n} \rightarrow \mathbb{R}, S:[0, T] \times \mathbb{R}^{n} \rightarrow \mathbb{R}^{m}, R:[0, T] \times \mathbb{R}^{n} \rightarrow \mathbb{S}^{m}$, and $G: \mathbb{R}^{n} \rightarrow \mathbb{R}$ are continuous. There are constants $L, Q_{0}, G_{0}, S_{0}>0, \varepsilon_{0} \in(0,1)$, and a continuous function $\rho: \mathbb{R}^{n} \rightarrow\left[\rho_{0}, \infty\right)$ with $\rho_{0}>0$ such that

$$
\left\{\begin{array}{l}
R(t, x) \geq \rho(x) I, \\
\left(1-\varepsilon_{0}\right) Q(t, x)-\frac{1}{2} S(t, x)^{T} R(t, x)^{-1} S(t, x), G(x) \geq-L, \\
Q(t, x) \leq Q_{0}\left(1+|x|^{2}\right), \quad G(x) \leq G_{0}\left(1+|x|^{2}\right), \quad|S(t, x)| \leq S_{0}(1+|x|), \\
\forall(t, x) \in[0, T] \times \mathbb{R}^{n},
\end{array}\right.
$$

and

$$
\left\{\begin{array}{l}
\left|B(t, x) R(t, x)^{-1} S(t, x)\right| \leq L(1+|x|), \quad(t, x) \in[0, T] \times \mathbb{R}^{n} . \\
\left|B(t, x) R(t, x)^{-1} B(t, x)^{T}\right| \leq L,
\end{array}\right.
$$

The first two conditions in $(2.7)$ will lead to the coercivity of the cost functional $u(\cdot) \mapsto J(t, x ; u(\cdot))$ and the last condition in (2.7) implies that our framework covers the standard LQ problem.

We also need the following assumption later.

(H3) The map $(t, x) \mapsto(A(t, x), B(t, x), Q(t, x), S(t, x), R(t, x), G(x))$ is twice continuously differentiable.

For any $0 \leq t<T$, let

$$
\mathcal{U}[t, T]=\left\{u(\cdot) \in L^{2}\left(t, T ; \mathbb{R}^{m}\right) \mid u(s) \in U, \quad \text { a.e. } s \in[t, T]\right\} .
$$

Any $u(\cdot) \in \mathcal{U}[t, T]$ is called an admissible control on $[t, T]$. We denote

$$
\|u(\cdot)\|_{L^{2}(t, s)}=\left(\int_{t}^{s}|u(r)|^{2} \mathrm{~d} r\right)^{\frac{1}{2}}, \quad \forall u(\cdot) \in \mathcal{U}[t, s] .
$$

The following simple result is concerned with the well-posedness of the state equation (1.1), whose proof is straightforward.

Proposition 2.1. Let (H1) hold. Then for any initial pair $(t, x) \in[0, T] \times \mathbb{R}^{n}$ and $u(\cdot) \in \mathcal{U}[t, T]$, state equation (1.1) admits a unique solution $X(\cdot) \equiv X(\cdot ; t, x, u(\cdot))$, and the following estimate holds:

$$
|X(s ; t, x, u(\cdot))| \leq K\left[1+|x|+\|u(\cdot)\|_{L^{2}(t, s)}\right], \quad \forall s \in[t, T],
$$

and

$$
|X(s ; t, x, u(\cdot))-x| \leq K\left[1+|x|+\|u(\cdot)\|_{L^{2}(t, s)}\right]\left[\sqrt{s-t}+\|u(\cdot)\|_{L^{2}(t, s)}\right] \sqrt{s-t},
$$

hereafter, $K>0$ denotes a generic constant which can be different from line to line. Further, for any $t \in[0, T]$, $x, \bar{x} \in \mathbb{R}^{n}$, and $u(\cdot) \in \mathcal{U}[t, T]$, it holds

$$
|X(s ; t, x, u(\cdot))-X(s ; t, \bar{x}, u(\cdot))| \leq \mathrm{e}^{\left(L_{A}+L_{B}\right)(T-t)}|x-\bar{x}|, \quad s \in[t, T] .
$$

In establishing the above estimates, condition (2.3) is used. As a consequence of the above, using the technique found in [16], we have the following result on the value function.

Proposition 2.2. Let $(\mathrm{H} 1)-(\mathrm{H} 2)$ hold. Then the value function $V(\cdot, \cdot)$ is continuous and there exists a constant $K>0$ such that

$$
-L(T-t+1) \leq V(t, x) \leq K\left(1+|x|^{2}\right), \quad \forall(t, x) \in[0, T] \times \mathbb{R}^{n},
$$

and

$$
|V(t, x)-V(t, \bar{x})| \leq K(|x| \vee|\bar{x}|)|x-\bar{x}|, \quad \forall t \in[0, T], x, \bar{x} \in \mathbb{R}^{n},
$$


where $|x| \vee|\bar{x}|=\max \{|x|,|\bar{x}|\}$. Moreover, the value function $V(\cdot, \cdot)$ is the unique viscosity solution to the following HJB equation:

$$
\left\{\begin{array}{l}
V_{t}(t, x)+\left\langle V_{x}(t, x), A(t, x)\right\rangle+Q(t, x) \\
\quad+\inf _{u \in U}\left[\left\langle B(t, x)^{T} V_{x}(t, x)+S(t, x), u\right\rangle+\frac{1}{2}\langle R(t, x) u, u\rangle\right]=0, \quad(t, x) \in[0, T] \times \mathbb{R}^{n}, \\
V(T, x)=G(x) .
\end{array}\right.
$$

Note that in the case $U=\mathbb{R}^{m}$, the above HJB equation can be written as

$$
\left\{\begin{array}{l}
V_{t}(t, x)+H\left(t, x, V_{x}(t, x)\right)=0, \quad(t, x) \in[0, T] \times \mathbb{R}^{n}, \\
V(T, x)=G(x), \quad x \in \mathbb{R}^{n}
\end{array}\right.
$$

with

$$
\begin{gathered}
H(t, x, p)=Q(t, x)+\langle p, A(t, x)\rangle-\frac{1}{2}\left[B(t, x)^{T} p+S(t, x)\right]^{T} R(t, x)^{-1}\left[B(t, x)^{T} p+S(t, x)\right], \\
(t, x, p) \in[0, T] \times \mathbb{R}^{n} \times \mathbb{R}^{n} .
\end{gathered}
$$

Further, in the case that $V(t, x)$ is differentiable, it is the classical solution to the above HJB equation and by the verification theorem, the optimal control admits the following state feedback representation:

$$
u(s)=-R(s, X(s))^{-1}\left[B(s, X(s))^{T} V_{x}(s, X(s))+S(s, X(s))\right], \quad s \in[t, T],
$$

with $X(\cdot)$ being the solution to the closed-loop system:

$$
\left\{\begin{array}{l}
\dot{X}(s)=A(s, X(s))-B(s, X(s)) R(s, X(s))^{-1}\left[B(s, X(s))^{T} V_{x}(s, X(s))+S(s, X(s))\right], \quad s \in[t, T], \\
X(t)=x .
\end{array}\right.
$$

Remark 2.3. Note that when $V(\cdot, \cdot)$ is differentiable, by $(2.14), x \mapsto V_{x}(s, x)$ is at most of linear growth. Therefore, by condition (2.8), the right hand side of equation in (2.19) is at most of linear growth in $X(s)$. Hence, the solution $X(\cdot)$ exists globally on $[t, T]$. Further, if $V(\cdot, \cdot)$ is twice continuously differentiable, then the right hand side of the equation in (2.19) is at least locally Lipschitz in $X(s)$, together with the linear growth in $X(s)$, we obtain the uniqueness of the solution $X(\cdot)$ to the closed-loop system (2.19).

From [16], we note that to guarantee the uniqueness of viscosity solution to the HJB equation, we need

$$
\left\{\begin{array}{l}
|H(t, x, p)-H(t, y, p)| \leq \omega(|x|+|y|,|p|,|x-y|), \quad t \in[0, T], x, y, p \in \mathbb{R}^{n}, \\
|H(t, x, p)-H(t, x, q)| \leq K_{0} \sum_{i=1}^{k}\langle x\rangle^{\lambda_{i}}(|p| \vee|q|)^{\nu_{i}}|p-q|, \quad t \in[0, T], x, p, q \in \mathbb{R}^{n}, \\
|G(x)-G(y)| \leq K_{0}(\langle x\rangle \vee\langle y\rangle)^{\mu-1}|x-y|, \quad \forall x, y \in \mathbb{R}^{n}, \\
\lambda_{i}, \nu_{i} \geq 0, \quad \lambda_{i}+(\mu-1) \nu_{i} \leq 1, \quad 1 \leq i \leq k,
\end{array}\right.
$$

with $\langle x\rangle=\sqrt{1+|x|^{2}}$. For the current case, we may let $\mu=2$. Then

$$
|G(x)-G(y)| \leq L(\langle x\rangle \vee\langle y\rangle)|x-y|, \quad \forall x, y \in \mathbb{R}^{n} .
$$

When $U=\mathbb{R}^{m}$, the Hamiltonian has the explicit form (2.17). Clearly, the first condition in (2.20) holds. For the second condition, we observe that

$$
\begin{aligned}
\left|H_{p}(t, x)\right| & \leq\left|A(t, x)+B(t, x) R(t, x)^{-1} S(t, x)\right|+\left|B(t, x) R(t, x)^{-1} B(t, x)^{T} p\right| \\
& \leq K_{0}(\langle x\rangle+|p|),
\end{aligned}
$$

which is implied by (2.8). Thus, the second condition in (2.20) holds with

$$
\lambda_{1}=\nu_{2}=1, \quad \lambda_{2}=\nu_{1}=0 .
$$




\section{Existence of optimal CONTROLS AND Minimum PRINCIPLE}

We first present the following result.

Proposition 3.1. Under (H1)-(H2), for any initial pair $(t, x) \in[0, T) \times \mathbb{R}^{n}$, Problem (AQ) admits an optimal control.

Proof. Let $(t, x) \in[0, T) \times \mathbb{R}^{n}$ be given. Let $X_{0}(\cdot)=X(\cdot ; t, x, 0)$. According to $(2.10)$, we have

$$
\left|X_{0}(s)\right| \leq K(1+|x|), \quad \forall s \in[t, T],
$$

for some $K>0$. Let $u^{k}(\cdot) \in \mathcal{U}[t, T]$ be a minimizing sequence with the corresponding state trajectory $X^{k}(\cdot) \equiv$ $X\left(\cdot ; t, x, u^{k}(\cdot)\right)$. Then we may assume that (making use of $(2.7)$ )

$$
\begin{aligned}
& J(t, x ; 0)+1 \geq J\left(t, x ; u^{k}(\cdot)\right) \\
& =\int_{t}^{T}\left[Q\left(s, X^{k}(s)\right)+\left\langle S\left(s, X^{k}(s)\right), u^{k}(s)\right\rangle+\frac{1}{2}\left\langle R\left(s, X^{k}(s)\right) u^{k}(s), u^{k}(s)\right\rangle\right] \mathrm{d} s+G\left(X^{k}(T)\right) \\
& =\int_{t}^{T}\left[\frac{1}{1-\varepsilon_{0}}\left(\left(1-\varepsilon_{0}\right) Q\left(s, X^{k}(s)\right)-\frac{1}{2} S\left(s, X^{k}(s)\right)^{T} R\left(s, X^{k}(s)\right)^{-1} S\left(s, X^{k}(s)\right)\right)\right. \\
& \quad+\frac{1}{2}\left|\left(1-\varepsilon_{0}\right)^{\frac{1}{2}} R\left(s, X^{k}(s)\right)^{\frac{1}{2}} u^{k}(s)+\left(1-\varepsilon_{0}\right)^{-\frac{1}{2}} R\left(s, X^{k}(s)\right)^{-\frac{1}{2}} S\left(s, X^{k}(s)\right)\right|^{2} \\
& \left.+\frac{\varepsilon_{0}}{2}\left\langle R\left(s, X^{k}(s)\right) u^{k}(s), u^{k}(s)\right\rangle\right] \mathrm{d} s+G\left(X^{k}(T)\right) \\
& \geq-\frac{L(T-t)}{1-\varepsilon_{0}}+\frac{\varepsilon_{0} \rho_{0}}{2} \int_{t}^{T}\left|u^{k}(s)\right|^{2} \mathrm{~d} s-L .
\end{aligned}
$$

Thus,

$$
\int_{t}^{T}\left|u^{k}(s)\right|^{2} \mathrm{~d} s \leq K, \quad \forall k \geq 1
$$

Consequently,

$$
\left|X^{k}(s)\right| \leq K\left(\left(1+|x|+\left\|u^{k}(\cdot)\right\|_{\mathcal{U}[t, T]}\right) \leq K, \quad \forall s \in[t, T], k \geq 1 .\right.
$$

Then for any $t \leq s<\tau \leq T$,

$$
\begin{aligned}
\left|X^{k}(\tau)-X^{k}(s)\right| & \left.\leq \int_{s}^{\tau}\left(A_{0}+L_{A}\left|X^{k}(r)\right|+\left(B_{0}+\widetilde{L}_{B} \mid X^{k}(r)\right) \mid\right)\left|u^{k}(r)\right|\right) \mathrm{d} r \\
& \leq K(\tau-s)+K(\tau-s)^{\frac{1}{2}}\left\|u^{k}(\cdot)\right\|_{\mathcal{U}[t, T]} \leq K(\tau-s)^{\frac{1}{2}} .
\end{aligned}
$$

Thus, $\left\{X^{k}(\cdot)\right\}$ is uniformly bounded and equicontinuous. Hence, we may assume that $X^{k}(\cdot) \rightarrow X^{*}(\cdot)$ in $C\left([t, T] ; \mathbb{R}^{n}\right)$. Then a standard argument applies to get the existence of an optimal control (see [7]).

The following is a kind of Pontryagin type minimum principle for Problem (AQ). The proof is pretty standard.

Proposition 3.2. Let $(\mathrm{H} 1)-(\mathrm{H} 3)$ hold and $(t, x) \in[0, T) \times \mathbb{R}^{n}$ be given. Let $\left(X^{*}(\cdot), u^{*}(\cdot)\right)$ be an optimal pair of Problem (AQ) for $(t, x)$. Then the following adjoint equation admits a unique solution

$$
\left\{\begin{aligned}
\dot{Y}(s)= & -\left[A_{x}\left(s, X^{*}(s)\right)+\sum_{j=1}^{m} u_{j}^{*}(s) B_{x}^{j}\left(s, X^{*}(s)\right)\right]^{T} Y(s)-Q_{x}\left(s, X^{*}(s)\right)^{T} \\
& -S_{x}\left(s, X^{*}(s)\right)^{T} u^{*}(s)-\frac{1}{2} \sum_{j, k=1}^{m} u_{j}^{*}(s) u_{k}^{*}(s) R_{x}^{j k}\left(s, X^{*}(s)\right)^{T}, \quad s \in[t, T], \\
Y(T)= & G_{x}\left(X^{*}(T)\right)^{T},
\end{aligned}\right.
$$


and the following minimum condition holds:

$$
\begin{aligned}
& {\left[B\left(s, X^{*}(s)\right)^{T} Y(s)+S\left(s, X^{*}(s)\right)\right] u^{*}(s)+\frac{1}{2} u^{*}(s)^{T} R\left(s, X^{*}(s)\right) u^{*}(s)} \\
& =\min _{u \in U}\left\{\left[B\left(s, X^{*}(s)\right)^{T} Y(s)+S\left(s, X^{*}(s)\right)\right] u+\frac{1}{2} u^{T} R\left(s, X^{*}(s)\right) u\right\}, \quad s \in[t, T] .
\end{aligned}
$$

In the above, $B(s, x)=\left(B^{1}(s, x), B^{2}(s, x), \cdots, B^{m}(s, x)\right)$ with $B^{i}:[0, T] \times \mathbb{R}^{n} \rightarrow \mathbb{R}^{n}$, and $B_{x}^{i}:[0, T] \times \mathbb{R}^{n} \rightarrow$ $\mathbb{R}^{n \times n}$. In particular, if $U=\mathbb{R}^{m}$, we have

$$
u^{*}(s)=-R\left(s, X^{*}(s)\right)^{-1}\left[B\left(s, X^{*}(s)\right)^{T} Y(s)+S\left(s, X^{*}(s)\right)\right], \quad s \in[t, T] .
$$

Hereafter, we assume that $U=\mathbb{R}^{m}$. From the above, we see that under $(\mathrm{H} 1)-(\mathrm{H} 3)$, for any $(t, x) \in[0, T) \times \mathbb{R}^{n}$, the following coupled two-point boundary value problem, called the optimality system of Problem (AQ), admits a solution $(X(\cdot), Y(\cdot))$ :

$$
\left\{\begin{aligned}
& \dot{X}(s)= A(s, X(s))-B(s, X(s)) R(s, X(s))^{-1}\left[B(s, X(s))^{T} Y(s)+S(s, X(s))\right] \\
& \dot{Y}(s)=- {\left[A_{x}(s, X(s))-\sum_{j=1}^{m} e_{j}^{T} R(s, X(s))^{-1}\left\{B(s, X(s))^{T} Y(s)+S(s, X(s))\right\} B_{x}^{j}(s, X(s))\right]^{T} Y(s) } \\
&-Q_{x}(s, X(s))^{T}+S_{x}(s, X(s))^{T} R(s, X(s))^{-1}\left[B(s, X(s))^{T} Y(s)+S(s, X(s))\right] \\
&-\frac{1}{2} \sum_{j, k=1}^{m}\left[B(s, X(s))^{T} Y(s)+S(s, X(s))\right]^{T} R(s, X(s))^{-1} e_{j} e_{k}^{T} R(s, X(s))^{-1} \\
& \cdot\left[B(s, X(s))^{T} Y(s)+S(s, X(s))\right] R_{x}^{j k}(s, X(s))^{T}, \quad s \in[t, T], \\
& X(t)=x, \quad Y(T)=G_{x}(X(T))^{T},
\end{aligned}\right.
$$

where $e_{j} \in \mathbb{R}^{m}$ is the vector with entry 1 at the $i$ th position and all other entries are zero. Further, if (3.6) admits a unique solution $(X(\cdot), Y(\cdot))$, then $X^{*}(\cdot)=X(\cdot)$ must be the optimal trajectory and the optimal control $u^{*}(\cdot)$ must be given by $(3.5)$.

We point out that optimal control $u^{*}(\cdot)$ of form (3.5) is not practically feasible because the involved $Y(\cdot)$ is obtained from the above optimality system (3.6), and some future information, say, $X(T)$ of the state trajectory $X(\cdot)$ seems to be needed. On the other hand, if we are able to show that actually one has

$$
Y(s)=\Theta(s, X(s)), \quad s \in[t, T]
$$

for some map $\Theta(\cdot, \cdot)$, then plugging the above into (3.5), we will end up with a nonlinear state feedback control which will be practically feasible, in principle. The efforts in the rest of this paper is trying to achieve such a goal, for the case $U=\mathbb{R}^{m}$. 


\section{Differentiability of the Value function}

Let us denote

$$
\left\{\begin{aligned}
& b(s, x, y)= A(s, x)-B(s, x) R(s, x)^{-1}\left[B(s, x)^{T} y+S(s, x)\right] \\
& g(s, x, y)=-\left[A_{x}(s, x)-\sum_{j=1}^{m} e_{j}^{T} R(s, x)^{-1}\left\{B(s, x)^{T} y+S(s, x)\right\} B_{x}^{j}(s, x)\right]^{T} y \\
&-Q_{x}(s, x)^{T}+S_{x}(s, x)^{T} R(s, x)^{-1}\left[B(s, x)^{T} y+S(s, x)\right] \\
&-\frac{1}{2} \sum_{j, k=1}^{m}\left[B(s, x)^{T} y+S(s, x)\right]^{T} R(s, x)^{-1} e_{j} e_{k}^{T} R(s, x)^{-1}\left[B(s, x)^{T} y+S(s, x)\right] R_{x}^{j k}(s, x)^{T}, \\
& h(x)=G_{x}(x)^{T}, \quad x \in \mathbb{R}^{n} . \quad(s, x) \in[0, T] \times \mathbb{R}^{n},
\end{aligned}\right.
$$

Then the optimality system (3.6) becomes

$$
\left\{\begin{array}{l}
\dot{X}(s)=b(s, X(s), Y(s)), \quad s \in[t, T], \\
\dot{Y}(s)=g(s, X(s), Y(s)), \\
X(t)=x, \quad Y(T)=h(X(T)) .
\end{array}\right.
$$

For such a two-point boundary value problem, we have the following result.

Proposition 4.1. Let (H1)-(H2) hold. Suppose further that there exists a constant $\lambda>0$ and a matrix $\Lambda \in$ $\mathbb{R}^{n \times n}$ such that

$$
\langle\Lambda(x-\bar{x}), h(x)-h(\bar{x})\rangle \geq \lambda|x-\bar{x}|^{2}, \quad \forall x, \bar{x} \in \mathbb{R}^{n},
$$

and

$$
\begin{gathered}
\left\langle\Lambda^{T}[g(s, x, y)-g(s, \bar{x}, \bar{y})], x-\bar{x}\right\rangle+\langle\Lambda[b(s, x, y)-b(s, \bar{x}, \bar{y})], y-\bar{y}\rangle \leq-\lambda|x-\bar{x}|^{2}, \\
\forall s \in[0, T], x, \bar{x}, y, \bar{y} \in \mathbb{R}^{n} .
\end{gathered}
$$

Then for any $(t, x) \in[0, T) \times \mathbb{R}^{n},(4.1)$ admits a unique solution $(X(\cdot), Y(\cdot))$. Consequently, Problem (AQ) admits a unique optimal control, the value function $V(\cdot, \cdot)$ is differentiable, and the optimal control admits a state feedback representation (2.18).

Proof. By regarding (4.1) as a special case of forward-backward stochastic differential equation, we may use the result of [13] (see also [15], p. 152, Prop. 3.4). Then the unique solvability of (4.1) follows. Since any optimal pair $\left(X^{*}(\cdot), u^{*}(\cdot)\right)$ of Problem (AQ) will lead to an optimality system of form (4.1), by the uniqueness, we see that the optimal pair must be unique. Then by [11], the value function must be differentiable. Finally, the state feedback representation (2.18) follows from a standard verification theorem.

When $b, g, h$ are differentiable, conditions (4.2)-(4.3) can further be written as

$$
h_{x}(x)^{T} \Lambda+\Lambda^{T} h_{x}(x) \geq 2 \lambda I, \quad \forall x \in \mathbb{R}^{n},
$$

and

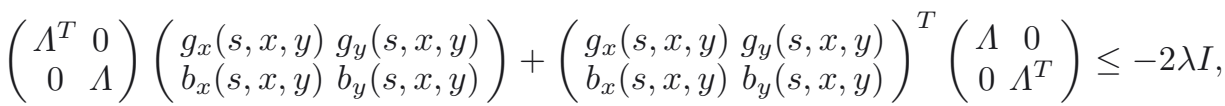

$$
\begin{aligned}
& \forall(s, x, y) \in[0, T] \times \mathbb{R}^{n} \times \mathbb{R}^{n} .
\end{aligned}
$$


Let us look at a simple situation for which the condition imposed above is pretty close to those we are familiar with. Consider the following linear state equation:

$$
\left\{\begin{array}{l}
\dot{X}(s)=A(s) X(s)+B(s) u(s), \quad s \in[t, T], \\
X(t)=x
\end{array}\right.
$$

with quadratic cost functional

$$
J(t, x ; u(\cdot))=\frac{1}{2} \int_{t}^{T}[\langle Q(s) X(s), X(s)\rangle+\langle R(s) u(s), u(s)\rangle] \mathrm{d} s+\frac{1}{2}\langle G X(T), X(T)\rangle .
$$

We assume that $R(\cdot)$ is uniformly positive definite. Then

$$
\left\{\begin{array}{l}
b(s, x, y)=A(s) x-B(s) R(s)^{-1} B(s)^{T} y, \\
g(s, x, y)=-A(s)^{T} y-Q(s) x .
\end{array}\right.
$$

Hence, by taking $\Lambda=I$, we see that (4.2) and (4.3) are equivalent to the following:

$$
G(s) \geq \lambda I, \quad Q(s)+B(s) R(s)^{-1} B(s)^{T} \geq \lambda I, \quad s \in[0, T],
$$

which are almost standard in the classical LQ problems.

We point out that (4.2) and (4.3) are basically some sort of monotonicity conditions. In fact, they mean that the maps $x \mapsto \Lambda^{T} h(x)$ and $(x, y) \mapsto-\left(\Lambda^{T} g(s, x, y), \Lambda b(s, x, y)\right)$ are uniformly monotone, in the sense of nonlinear monotone operators [25]. For the unique solvability of system (3.6), one may impose some other type conditions. See for examples, $[15,18,20]$, and so on. Therefore, there are some other situations, besides the case in the above proposition, for which optimal control of Problem (AQ) uniquely exists and it has a state feedback representation. However, the above-mentioned conditions usually will look very complicated, and practically, they are not easy to use. Hence, some other alternative approaches to Problem (AQ) are also desirable.

Note that in the case $U=\mathbb{R}^{m}$, for any $t \in[0, T), \mathcal{U}[t, T]$ is a Hilbert space whose dual $\mathcal{U}[t, T]^{*}$ can be identified with $\mathcal{U}[t, T]$ by the Riesz representation theorem. For any initial pair $(t, x) \in \mathcal{U}[t, T]$, by Proposition 3.1 , under (H1)-(H2), Problem (AQ) admits an optimal control $u^{*}(\cdot) \in \mathcal{U}[t, T]$. Thus, under (H1)-(H3), we have

$$
D_{u} J\left(t, x ; u^{*}(\cdot)\right)=0,
$$

and

$$
D_{u u} J\left(t, x ; u^{*}(\cdot)\right) \geq 0
$$

i.e., $D_{u u} J\left(t, x ; u^{*}(\cdot)\right)$ is positive semi-definite, meaning that

$$
\left\langle D_{u u} J\left(t, x ; u^{*}(\cdot)\right) v(\cdot), v(\cdot)\right\rangle \geq 0, \quad \forall v(\cdot) \in \mathcal{U}[t, T] .
$$

Now, suppose the above is strengthened to the following:

$$
\left\langle D_{u u} J(t, x ; u(\cdot)) v(\cdot), v(\cdot)\right\rangle \geq \delta\|v(\cdot)\|_{L^{2}(t, T)}^{2}, \quad \forall u(\cdot), v(\cdot) \in \mathcal{U}[t, T],
$$

for some $\delta>0$, then $u(\cdot) \mapsto J(t, x ; u(\cdot))$ is uniformly convex, which implies that Problem (AQ) admits a unique optimal control for any initial pair $(t, x) \in[0, T) \times \mathbb{R}^{n}$. Hence, the value function $V(\cdot, \cdot)$ is differentiable. The following result says that under (4.8), we actually have a little more.

Theorem 4.2. Let (H1)-(H3) hold and (4.8) be satisfied for some $\delta>0$. Then the optimal control map $(t, x) \mapsto$ $u^{*}(\cdot ; t, x)$ is differentiable and the value function $V(\cdot, \cdot)$ is twice differentiable. 
Proof. First of all, we naturally extend $u(\cdot) \mapsto J(t, x ; u(\cdot))$ from $\mathcal{U}[t, T]$ to $\mathcal{U}[0, T]$ as follows:

$$
\widehat{J}(t, x ; u(\cdot))=\frac{\delta}{2} \int_{0}^{t}|u(s)|^{2} \mathrm{~d} s+J\left(t, x ;\left.u(\cdot)\right|_{[t, T]}\right), \quad \forall u(\cdot) \in \mathcal{U}[0, T],
$$

where $\left.u(\cdot)\right|_{[t, T]}$ stands for the restriction of $u(\cdot) \in \mathcal{U}[0, T]$ on $[t, T]$. Then for any $(t, x, u(\cdot)) \in[0, T] \times \mathbb{R}^{n} \times \mathcal{U}[0, T]$,

$$
\left\langle D_{u} \widehat{J}(t, x ; u(\cdot)), v(\cdot)\right\rangle=\delta \int_{0}^{t}\langle u(s), v(s)\rangle \mathrm{d} s+\left\langle D_{u} J(t, x ; u(\cdot)),\left.v(\cdot)\right|_{[t, T]}\right\rangle, \quad \forall v(\cdot) \in \mathcal{U}[0, T],
$$

and

$$
\begin{aligned}
& \left\langle\left[D_{u u} \widehat{J}(t, x ; u(\cdot))\right] v(\cdot), w(\cdot)\right\rangle=\delta \int_{0}^{t}\langle v(s), w(s)\rangle \mathrm{d} s \\
& +\left\langle\left.\left[D_{u u} J\left(t, x ;\left.u(\cdot)\right|_{[t, T]}\right)\right] v(\cdot)\right|_{[t, T]},\left.w(\cdot)\right|_{[t, T]}\right\rangle, \quad \forall v(\cdot), w(\cdot) \in \mathcal{U}[0, T] .
\end{aligned}
$$

Further, $\widehat{u}^{*}(\cdot)$ is a minimum of $u(\cdot) \mapsto \widehat{J}(t, x ; u(\cdot))$ over $\mathcal{U}[0, T]$ if and only if

$$
\widehat{u}^{*}(s)=u^{*}(s) I_{[t, T]}(s), \quad s \in[0, T],
$$

with $u^{*}(\cdot)$ being a minimum of $u(\cdot) \mapsto J(t, x ; u(\cdot))$ over $\mathcal{U}[t, T]$. Clearly, by (4.8), we see that

$$
\left\langle D_{u u} \widehat{J}(t, x ; u(\cdot)) v(\cdot), v(\cdot)\right\rangle \geq \delta\|v(\cdot)\|_{L^{2}(0, T)}^{2}, \quad \forall u(\cdot), v(\cdot) \in \mathcal{U}[0, T] .
$$

Thus, for any $(t, x) \in[0, T) \times \mathbb{R}^{n}$, the map $u(\cdot) \mapsto \widehat{J}(t, x ; u(\cdot))$ is uniformly convex and therefore, it admits a unique minimum $\widehat{u}^{*}(\cdot) \in \mathcal{U}[0, T]$. Now, let us define

$$
F(t, x, u(\cdot))=D_{u} \widehat{J}(t, x ; u(\cdot)), \quad \forall(t, x, u(\cdot)) \in[0, T] \times \mathbb{R}^{n} \times \mathcal{U}[0, T] .
$$

Then $F:[0, T] \times \mathbb{R}^{n} \times \mathcal{U}[0, T] \rightarrow \mathcal{U}[0, T]^{*}=\mathcal{U}[0, T]$. For any fixed initial pair $(t, x) \in[0, T] \times \mathbb{R}^{n}$, consider the following equation:

$$
F(t, x, u(\cdot))=0 .
$$

Under (H1)-(H3), from Proposition 3.1, and (4.8), $u(\cdot) \mapsto \widehat{J}(t, x ; u(\cdot))$ admits a unique minimum $\widehat{u}^{*}(\cdot) \equiv$ $\widehat{u}^{*}(\cdot ; t, x) \in \mathcal{U}[0, T]$. Thus,

$$
V(t, x)=\inf _{u(\cdot) \in \mathcal{U}[t, T]} J(t, x ; u(\cdot))=J\left(t, x ;\left.\widehat{u}^{*}(\cdot)\right|_{[t, T]}\right)=\widehat{J}\left(t, x ; \widehat{u}^{*}(\cdot)\right)=\inf _{u(\cdot) \in \mathcal{U}[0, T]} \widehat{J}(t, x ; u(\cdot)) .
$$

Then it is necessary that $\widehat{u}^{*}(\cdot)$ is a solution to equation (4.10), and

$$
F_{u}\left(t, x ; \widehat{u}^{*}(\cdot)\right)=D_{u u} \widehat{J}\left(t, x ; \widehat{u}^{*}(\cdot)\right) \geq \delta I .
$$

This implies that $F_{u}\left(t, x ; \widehat{u}^{*}(\cdot)\right)^{-1}: \mathcal{U}[0, T] \rightarrow \mathcal{U}[0, T]$ exists and is a bounded operator ([8], Lem. 4.123 on p. 365). Then, by implicit function Theorem ([12], see also [24], Thm. 4.B on pp. 150-151), we have a differentiable map $(s, y) \mapsto \varphi(\cdot ; s, y)$ defined in an open ball $\mathcal{O}_{\varepsilon}(t, x)$ centered at $(t, x)$ with radius $\varepsilon>0$ such that

$$
\left\{\begin{array}{l}
\varphi(\cdot ; t, x)=\widehat{u}^{*}(\cdot ; t, x), \\
F(s, y ; \varphi(\cdot ; s, y)) \equiv D_{u} \widehat{J}(s, y ; \varphi(\cdot ; s, y))=0, \quad(s, y) \in \mathcal{O}_{\varepsilon}(t, x), \\
F_{u}(s, y ; \varphi(\cdot ; s, y)) \equiv D_{u u} J(s, y ; \varphi(\cdot ; s, y)) \geq \delta I, \quad(s, y) \in \mathcal{O}_{\varepsilon}(t, x) .
\end{array}\right.
$$

and at $(t, x)$,

$$
\begin{aligned}
\varphi_{(t, x)}(\cdot ; t, x) & =F_{u}\left(t, x ; \widehat{u}^{*}(\cdot)\right)^{-1} F_{(t, x)}\left(t, x ; \widehat{u}^{*}(\cdot)\right) \\
& \equiv-D_{u u} \widehat{J}\left(t, x ; u^{*}(\cdot ; t, x)\right)^{-1}\left[D_{u} \widehat{J}\right]_{(t, x)}\left(t, x ; \widehat{u}^{*}(\cdot ; t, x)\right) .
\end{aligned}
$$


From (4.13), we see that for any $(s, y) \in \mathcal{O}_{\varepsilon}(t, x), \varphi(\cdot ; s, y)$ must be a local minimum of $\widehat{J}(s, y ; u(\cdot))$. By the convexity of the functional $u(\cdot) \mapsto \widehat{J}(s, y ; u(\cdot))$, this local minimum must be global. Thus, by the uniqueness of the optimal control, we actually have

$$
\varphi(\cdot ; s, y)=\widehat{u}^{*}(\cdot ; s, y), \quad \forall(s, y) \in \mathcal{O}_{\varepsilon}(t, x) .
$$

Hence, $(t, x) \mapsto \widehat{u}^{*}(\cdot ; t, x)$ is continuously differentiable. Next, denoting $x_{0}=t$, we have

$$
V\left(x_{0}, x\right)=\widehat{J}\left(x_{0}, x ; \widehat{u}^{*}\left(\cdot ; x_{0}, x\right)\right), \quad D_{u} \widehat{J}\left(x_{0}, x ; \widehat{u}^{*}\left(\cdot ; x_{0}, x\right)\right)=0 .
$$

Thus, for $0 \leq i \leq n$,

$$
\widehat{u}_{x_{i}}^{*}\left(\cdot ; x_{0}, x\right)=-D_{u u} \widehat{J}\left(x_{0}, x ; \widehat{u}^{*}\left(\cdot ; x_{0}, x\right)\right)^{-1} D_{u} \widehat{J}_{x_{i}}\left(x_{0}, x ; \widehat{u}^{*}\left(\cdot ; x_{0}, x\right)\right) .
$$

Hence,

$$
V_{x_{i}}\left(x_{0}, x\right)=\widehat{J}_{x_{i}}\left(x_{0}, x ; \widehat{u}^{*}\left(\cdot ; x_{0}, x\right)\right)+D_{u} \widehat{J}\left(x_{0}, x ; \widehat{u}^{*}\left(\cdot ; x_{0}, x\right)\right) \widehat{u}_{x_{i}}^{*}\left(\cdot ; x_{0}, x\right)=\widehat{J}_{x_{i}}\left(x_{0}, x ; \widehat{u}^{*}\left(\cdot ; x_{0}, x\right)\right) .
$$

Further, for $0 \leq i, j \leq n$,

$$
\begin{aligned}
V_{x_{i} x_{j}}\left(x_{0}, x\right)= & \widehat{J}_{x_{i} x_{j}}\left(x_{0}, x ; \widehat{u}^{*}\left(\cdot ; x_{0}, x\right)\right)+D_{u} \widehat{J}_{x_{i}}\left(x_{0}, x ; \widehat{u}^{*}\left(\cdot ; x_{0}, x\right)\right) \widehat{u}_{x_{j}}^{*}\left(\cdot ; x_{0}, x\right) \\
& +\left[D_{u u} \widehat{J}\left(x_{0}, x ; u^{*}\left(\cdot ; x_{0}, x\right)\right) \widehat{u}_{x_{i}}^{*}\left(\cdot ; x_{0}, x\right)\right] \widehat{u}_{x_{j}}^{*}\left(\cdot ; x_{0}, x\right) \\
= & \widehat{J}_{x_{i} x_{j}}\left(x_{0}, x ; \widehat{u}^{*}\left(\cdot ; x_{0}, x\right)\right)+\left[D_{u u} \widehat{J}\left(x_{0}, x ; u^{*}\left(\cdot ; x_{0}, x\right)\right) \widehat{u}_{x_{i}}^{*}\left(\cdot ; x_{0}, x\right)\right] \widehat{u}_{x_{j}}^{*}\left(\cdot ; x_{0}, x\right) .
\end{aligned}
$$

Therefore, $V(\cdot, \cdot)$ is twice continuously differentiable.

\section{FréChet Differentiability of the Cost funCtional}

In this section, we will calculate the first and the second order Fréchet derivatives of the map $u(\cdot) \mapsto$ $J(t, x ; u(\cdot))$. Then we will find sufficient conditions for (4.8) to be true. Denote

$$
x=\left(\begin{array}{c}
x^{1} \\
x^{2} \\
\vdots \\
x^{n}
\end{array}\right), y=\left(\begin{array}{c}
y^{1} \\
y^{2} \\
\vdots \\
y^{n}
\end{array}\right) \in \mathbb{R}^{n}, \quad u=\left(\begin{array}{c}
u^{1} \\
u^{2} \\
\vdots \\
u^{m}
\end{array}\right) \in \mathbb{R}^{m},
$$

and

$$
\begin{gathered}
A(t, x)=\left(\begin{array}{c}
A^{1}(t, x) \\
A^{2}(t, x) \\
\vdots \\
A^{n}(t, x)
\end{array}\right), \quad B(t, x)=\left(\begin{array}{cccc}
B^{11}(t, x) & B^{12}(t, x) & \cdots & B^{1 m}(t, x) \\
B^{21}(t, x) & B^{22}(t, x) & \cdots & B^{2 m}(t, x) \\
\vdots & \vdots & \ddots & \vdots \\
B^{n 1}(t, x) & B^{n 2}(t, x) & \cdots & B^{n m}(t, x)
\end{array}\right), \\
S(t, x)=\left(\begin{array}{c}
S^{1}(t, x) \\
S^{2}(t, x) \\
\vdots \\
S^{m}(t, x)
\end{array}\right), \quad R(t, x)=\left(\begin{array}{cccc}
R^{11}(t, x) & R^{12}(t, x) & \cdots & R^{1 m}(t, x) \\
R^{21}(t, x) & R^{22}(t, x) & \cdots & R^{2 m}(t, x) \\
\vdots & \vdots & \ddots & \vdots \\
R^{m 1}(t, x) & R^{m 2}(t, x) & \cdots & R^{m m}(t, x)
\end{array}\right), \\
A^{i}, B^{i j}, S^{j}, R^{j k}:[0, T] \times \mathbb{R}^{n} \rightarrow \mathbb{R}, \quad \begin{array}{c}
1 \leq n, 1 \leq j, k \leq m .
\end{array}
\end{gathered}
$$


Next, we denote

$$
\begin{aligned}
& B^{j}(t, x)=\left(\begin{array}{c}
B^{1 j}(t, x) \\
B^{2 j}(t, x) \\
\vdots \\
B^{n j}(t, x)
\end{array}\right), \widetilde{B}^{i}(t, x)=\left(\begin{array}{c}
B^{i 1}(t, x) \\
B^{i 2}(t, x) \\
\vdots \\
B^{i m}(t, x)
\end{array}\right), \\
& \forall(t, x) \in[0, T] \times \mathbb{R}^{n}, 1 \leq j \leq m, 1 \leq i \leq n .
\end{aligned}
$$

Then,

$$
\left\{\begin{array}{l}
B(t, x)=\left(B^{1}(t, x), B^{2}(t, x), \cdots, B^{m}(t, x)\right), \\
B(t, x)^{T}=\left(\widetilde{B}^{1}(t, x), \widetilde{B}^{2}(t, x), \cdots, \widetilde{B}^{n}(t, x)\right),
\end{array} \quad(t, x) \in[0, T] \times \mathbb{R}^{n} .\right.
$$

We have the following result.

Theorem 5.1. Let (H1)-(H3) hold. Then for any $(t, x) \in[0, T) \times \mathbb{R}^{n}$ and $u(\cdot) \in \mathcal{U}[t, T]$,

$$
\left\langle D_{u} J(t, x ; u(\cdot)), v(\cdot)\right\rangle=\int_{t}^{T}\left[R(s, X(s)) u(s)+S(s, X(s))+B(s, X(s))^{T} Y(s)\right] v(s) \mathrm{d} s, \quad \forall v(\cdot) \in \mathcal{U}[t, T] .
$$

with $(X(\cdot), Y(\cdot))$ being the solution to the following decoupled two-point boundary value problem:

$$
\left\{\begin{aligned}
\dot{X}(s)= & A(s, X(s))+B(s, X(s)) u(s) \\
\dot{Y}(s)= & -\left[A_{x}(s, X(s))+\sum_{j=1}^{m} u^{j}(s) B_{x}^{j}(s, X(s))\right]^{T} Y(s)-Q_{x}(s, X(s))^{T} \\
& -S_{x}(s, X(s))^{T} u(s)-\frac{1}{2} \sum_{j, k=1}^{m} u^{j}(s) u^{k}(s) R_{x}^{j k}(s, X(s))^{T} \\
X(t)= & x, \quad Y(T)=G_{x}(X(T))^{T}
\end{aligned}\right.
$$

Further,

$$
\begin{aligned}
\left\langle\left[D_{u u} J(t, x ; u(\cdot)) v(\cdot)\right], w(\cdot)\right\rangle=\int_{t}^{T}\left[R(s, X(s)) v(s)+B(s, X(s))^{T} Y_{1}(s)+\mathbf{C}(s) X_{1}(s)\right] w(s) \mathrm{d} s, & \forall v(\cdot), w(\cdot) \in \mathcal{U}[t, T]
\end{aligned}
$$

where

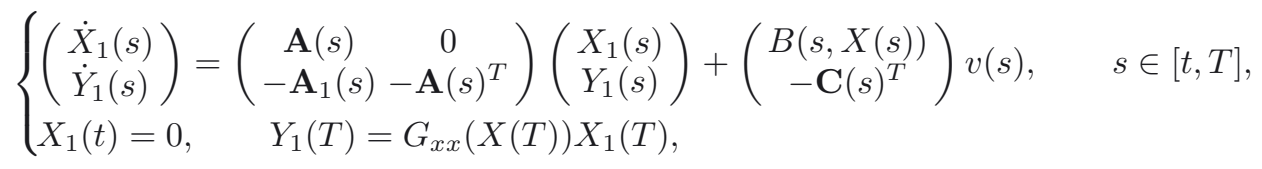

with

$$
\left\{\begin{aligned}
\mathbf{A}(s)= & A_{x}(s, X(s))+\sum_{j=1}^{m} u^{j}(s) B_{x}^{j}(s, X(s)), \\
\mathbf{A}_{1}(s)= & \sum_{i=1}^{n} Y^{i}(s) A_{x x}^{i}(s, X(s))+Q_{x x}(s, X(s))+\frac{1}{2} \sum_{j, k=1}^{m} u^{j}(s) u^{k}(s) R_{x x}^{j k}(s, X(s)) \\
& +\sum_{j=1}^{m} u^{j}(s)\left[\sum_{i=1}^{n} Y^{i}(s) B_{x x}^{i j}(s, X(s))+S_{x x}^{j}(s, X(s))\right] \\
\mathbf{C}(s)= & \sum_{j=1}^{m} u^{j}(s) R_{x}^{j}(s, X(s))+S_{x}(s, X(s))+\sum_{i=1}^{n} Y^{i}(s) \widetilde{B}_{x}^{i}(s, X(s)) .
\end{aligned}\right.
$$


Proof. Let $(t, x) \in[0, T) \times \mathbb{R}^{n}$ be fixed and $u(\cdot), v(\cdot) \in \mathcal{U}[t, T]$, let

$$
X(\cdot)=X(\cdot ; t, x, u(\cdot)), \quad X^{\varepsilon}(\cdot)=X(\cdot ; t, x, u(\cdot)+\varepsilon v(\cdot)),
$$

with $\varepsilon>0$. Let

$$
X_{1}(\cdot)=\lim _{\varepsilon \rightarrow 0} \frac{X^{\varepsilon}(\cdot)-X(\cdot)}{\varepsilon}
$$

Then

$$
\begin{aligned}
\dot{X}_{1}(s) & =\lim _{\varepsilon \rightarrow 0}\left\{\frac{A\left(s, X^{\varepsilon}(s)\right)-A(s, X(s))}{\varepsilon}+\sum_{j=1}^{m} u^{j}(s) \frac{B^{j}\left(s, X^{\varepsilon}(s)\right)-B^{j}(s, X(s))}{\varepsilon}\right\}+B(s, X(s)) v(s) \\
& =\left[A_{x}(s, X(s))+\sum_{j=1}^{m} u^{j}(s) B_{x}^{j}(s, X(s))\right] X_{1}(s)+B(s, X(s)) v(s) .
\end{aligned}
$$

Thus, $X_{1}(\cdot)$ solves the following:

$$
\left\{\begin{array}{l}
\dot{X}_{1}(s)=\mathbf{A}(s) X_{1}(s)+B(s, X(s)) v(s), \quad s \in[t, T], \\
X_{1}(t)=0
\end{array}\right.
$$

with $\mathbf{A}(\cdot)$ being defined in (5.5). We have

$$
\begin{aligned}
& \left\langle D_{u} J(t, x ; u(\cdot)), v(\cdot)\right\rangle=\lim _{\varepsilon \rightarrow 0} \frac{J(t, x ; u(\cdot)+\varepsilon v(\cdot))-J(t, x ; u(\cdot))}{\varepsilon} \\
& =\int_{t}^{T}\left[Q_{x}(s, X(s)) X_{1}(s)+\langle S(s, X(s)), v(s)\rangle+\left\langle S_{x}(s, X(s)) X_{1}(s), u(s)\right\rangle\right. \\
& \left.\quad+\langle R(s, X(s)) u(s), v(s)\rangle+\frac{1}{2} \sum_{j, k=1}^{m} u^{j}(s) u^{k}(s) R_{x}^{j k}(s, X(s)) X_{1}(s)\right] \mathrm{d} s+G_{x}(X(T)) X_{1}(T) \\
& =\int_{t}^{T}\left[\left\langle Q_{x}(s, X(s))^{T}+S_{x}(s, X(s))^{T} u(s)+\frac{1}{2} \sum_{j, k=1}^{m} u^{j}(s) u^{k}(s) R_{x}^{j k}(s, X(s))^{T}, X_{1}(s)\right\rangle\right. \\
& \quad+\langle S(s, X(s))+R(s, X(s)) u(s), v(s)\rangle] \mathrm{d} s+G_{x}(X(T)) X_{1}(T) .
\end{aligned}
$$

Let $(X(\cdot), Y(\cdot))$ be the solution to $(5.2)$. Then (note (5.5))

$$
\begin{aligned}
& \frac{\mathrm{d}}{\mathrm{d} s}\left\langle Y(s), X_{1}(s)\right\rangle=\left\langle\dot{Y}(s), X_{1}(s)\right\rangle+\left\langle Y(s), \mathbf{A}(s) X_{1}(s)\right\rangle+\langle Y(s), B(s, X(s)) v(s)\rangle \\
& =\left\langle\dot{Y}(s)+\mathbf{A}(s)^{T} Y(s), X_{1}(s)\right\rangle+\left\langle B(s, X(s))^{T} Y(s), v(s)\right\rangle .
\end{aligned}
$$

Noting $X_{1}(t)=0$, one has

$$
\begin{aligned}
G_{x}(X(T)) X_{1}(T) & =\left\langle Y(T), X_{1}(T)\right\rangle \\
& =\int_{t}^{T}\left\{\left\langle\dot{Y}(s)+\mathbf{A}(s)^{T} Y(s), X_{1}(s)\right\rangle+\left\langle B(s, X(s))^{T} Y(s), v(s)\right\rangle\right\} \mathrm{d} s .
\end{aligned}
$$


Consequently,

$$
\begin{aligned}
& \left\langle D_{u} J(t, x ; u(\cdot)), v(\cdot)\right\rangle=\int_{t}^{T}\left\{\left\langle\dot{Y}(s)+\mathbf{A}(s)^{T} Y(s)\right.\right. \\
& \left.\quad+Q_{x}(s, X(s))^{T}+S_{x}(s, X(s))^{T} u(s)+\frac{1}{2} \sum_{j, k=1}^{m} u^{j}(s) u^{k}(s) R_{x}^{j k}(s, X(s))^{T}, X_{1}(s)\right\rangle \\
& \left.\quad+\left\langle B(s, X(s))^{T} Y(s)+S(s, X(s))+R(s, X(s)) u(s), v(s)\right\rangle\right\} \mathrm{d} s \\
& =\int_{t}^{T}\left\langle R(s, X(s)) u(s)+S(s, X(s))+B(s, X(s))^{T} Y(s), v(s)\right\rangle \mathrm{d} s .
\end{aligned}
$$

This proves (5.1).

Next, we calculate $D_{u u} J(t, x ; u(\cdot))$. To this end, for any $\varepsilon \in(0,1)$, let $\left(X^{\varepsilon}(\cdot), Y^{\varepsilon}(\cdot)\right)$ be the solution to the following:

$$
\left\{\begin{aligned}
\dot{X}^{\varepsilon}(s)= & A\left(s, X^{\varepsilon}(s)\right)+B\left(s, X^{\varepsilon}(s)\right)[u(s)+\varepsilon v(s)], \\
\dot{Y}^{\varepsilon}(s)= & -\left[A_{x}\left(s, X^{\varepsilon}(s)\right)+\sum_{j=1}^{m}\left[u^{j}(s)+\varepsilon v^{j}(s)\right] B_{x}^{j}\left(s, X^{\varepsilon}(s)\right)\right]^{T} Y^{\varepsilon}(s) \\
& -Q_{x}\left(s, X^{\varepsilon}(s)\right)^{T}-S_{x}\left(s, X^{\varepsilon}(s)\right)^{T}[u(s)+\varepsilon v(s)] \\
& -\frac{1}{2} \sum_{j, k=1}^{m}\left[u^{j}(s)+\varepsilon v^{j}(s)\right]\left[u^{k}(s)+\varepsilon v^{k}(s)\right] R_{x}^{j k}\left(s, X^{\varepsilon}(s)\right)^{T}, \\
X^{\varepsilon}(t)= & x, \quad Y^{\varepsilon}(T)=G_{x}\left(X^{\varepsilon}(T)\right)^{T}
\end{aligned}\right.
$$

Then

$$
\begin{gathered}
{\left[D_{u} J(t, x ; u(\cdot)+\varepsilon v(\cdot))\right](s)=R\left(s, X^{\varepsilon}(s)\right)[u(s)+\varepsilon v(s)]+S\left(s, X^{\varepsilon}(s)\right)+B\left(s, X^{\varepsilon}(s)\right)^{T} Y^{\varepsilon}(s),} \\
s \in[t, T] .
\end{gathered}
$$

Hence,

$$
\begin{aligned}
& {\left[D_{u u} J(t, x ; u(\cdot)) v(\cdot)\right](s)=\lim _{\varepsilon \rightarrow 0} \frac{\left[D_{u} J(t, x ; u(\cdot)+\varepsilon v(\cdot))\right](s)-\left[D_{u} J(t, x ; u(\cdot))\right](s)}{\varepsilon} } \\
&=\lim _{\varepsilon \rightarrow 0}\left\{R\left(s, X^{\varepsilon}(s)\right) v(s)+\frac{R\left(s, X^{\varepsilon}(s)\right)-R(s, X(s))}{\varepsilon} u(s)+\frac{S\left(x, X^{\varepsilon}(s)\right)-S(s, X(s))}{\varepsilon}\right. \\
&\left.\quad+B\left(s, X^{\varepsilon}(s)\right)^{T} \frac{Y^{\varepsilon}(s)-Y(s)}{\varepsilon}+\frac{B\left(s, X^{\varepsilon}(s)\right)^{T}-B(s, X(s))^{T}}{\varepsilon} Y(s)\right\} \\
&=R(s, X(s)) v(s)+\sum_{j=1}^{m} u^{j}(s) R_{x}^{j}(s, X(s)) X_{1}(s)+S_{x}(s, X(s)) X_{1}(s) \\
& \quad+B(s, X(s))^{T} Y_{1}(s)+\sum_{i=1}^{n} Y^{i}(s) \widetilde{B}_{x}^{i}(s, X(s)) X_{1}(s) \\
&=R(s, X(s)) v(s)+B(s, X(s))^{T} Y_{1}(s) \\
& \quad+\left[\sum_{j=1}^{m} u^{j}(s) R_{x}^{j}(s, X(s))+S_{x}(s, X(s))+\sum_{i=1}^{n} Y^{i}(s) \widetilde{B}_{x}^{i}(s, X(s))\right] X_{1}(s) \\
& \equiv R(s, X(s)) v(s)+B(s, X(s))^{T} Y_{1}(s)+\mathbf{C}(s) X_{1}(s),
\end{aligned}
$$


where

$$
Y_{1}(s)=\lim _{\varepsilon \rightarrow 0} \frac{Y^{\varepsilon}(s)-Y(s)}{\varepsilon},
$$

and $\mathbf{C}(\cdot)$ is defined in (5.5). Then to complete the proof, we need only to derive the equation for $Y_{1}(\cdot)$. First of all,

$$
\begin{aligned}
Y_{1}(T) & =\lim _{\varepsilon \rightarrow 0} \frac{Y^{\varepsilon}(T)-Y(T)}{\varepsilon}=\lim _{\varepsilon \rightarrow 0} \frac{G_{x}\left(X^{\varepsilon}(T)\right)^{T}-G_{x}(X(T))^{T}}{\varepsilon} \\
& =G_{x x}(X(T))\left[\lim _{\varepsilon \rightarrow 0} \frac{X^{\varepsilon}(T)-X(T)}{\varepsilon}\right]=G_{x x}(X(T)) X_{1}(T) .
\end{aligned}
$$

Next,

$$
\begin{aligned}
\dot{Y}_{1}(s)= & \lim _{\varepsilon \rightarrow 0} \frac{\dot{Y}^{\varepsilon}(s)-\dot{Y}(s)}{\varepsilon} \\
= & -\lim _{\varepsilon \rightarrow 0}\left\{A_{x}\left(s, X^{\varepsilon}(s)\right)^{T} \frac{Y^{\varepsilon}(s)-Y(s)}{\varepsilon}+\sum_{i=1}^{n} Y^{i} \frac{A_{x}^{i}\left(s, X^{\varepsilon}(s)\right)^{T}-A_{x}^{i}(s, X(s))^{T}}{\varepsilon}\right. \\
& +\sum_{j=1}^{m} v^{j}(s) B_{x}^{j}\left(s, X^{\varepsilon}(s)\right)^{T} Y^{\varepsilon}(s)+\sum_{j=1}^{m} u^{j}(s) B_{x}^{j}\left(s, X^{\varepsilon}(s)\right)^{T} \frac{Y^{\varepsilon}(s)-Y(s)}{\varepsilon} \\
& +\sum_{j=1}^{m} \sum_{i=1}^{n} u^{j}(s) \frac{B_{x}^{i j}\left(s, X^{\varepsilon}(s)\right)^{T}-B_{x}^{i j}(s, X(s))^{T}}{\varepsilon} Y^{i}(s)+\frac{Q_{x}\left(X^{\varepsilon}(s)\right)^{T}-Q_{x}(X(s))^{T}}{\varepsilon} \\
& +S_{x}\left(s, X^{\varepsilon}(s)\right)^{T} v(s)+\frac{S_{x}\left(s, X^{\varepsilon}(s)\right)^{T}-S_{x}(s, X(s))^{T}}{\varepsilon} u(s) \\
& \left.+\frac{1}{2} \sum_{j, k=1}^{m} u^{j}(s) u^{k}(s) \frac{R_{x}^{j k}\left(s, X^{\varepsilon}(s)\right)^{T}-R_{x}^{j k}(s, X(s))^{T}}{\varepsilon}+\sum_{j, k=1}^{m} u^{j}(s) v^{k}(s) R_{x}^{j k}\left(s, X^{\varepsilon}(s)\right)^{T}\right\} .
\end{aligned}
$$

Hence,

$$
\begin{aligned}
\dot{Y}_{1}(s)= & -A_{x}(s, X(s))^{T} Y_{1}(s)-\sum_{i=1}^{n} Y^{i}(s) A_{x x}^{i}(s, X(s)) X_{1}(s)-\sum_{j=1}^{m} v^{j}(s) B_{x}^{j}(s, X(s))^{T} Y(s) \\
& -\sum_{j=1}^{m} u^{j}(s) B_{x}^{j}(s, X(s))^{T} Y_{1}(s)-\sum_{j=1}^{m} \sum_{i=1}^{n} u^{j}(s) Y^{i}(s) B_{x x}^{i j}(s, X(s)) X_{1}(s) \\
& -Q_{x x}(s, X(s)) X_{1}(s)-S_{x}(s, X(s))^{T} v(s)-\sum_{j=1}^{m} u^{j}(s) S_{x x}^{j}(s, X(s)) X_{1}(s) \\
& -\frac{1}{2} \sum_{j, k=1}^{m} u^{j}(s) u^{k}(s) R_{x x}^{j k}(s, X(s)) X_{1}(s)-\sum_{j, k=1}^{m} u^{j}(s) v^{k}(s) R_{x}^{j k}(s, X(s))^{T} \\
= & -\left[A_{x}(s, X(s))+\sum_{j=1}^{m} u^{j}(s) B_{x}^{j}(s, X(s))\right]^{T} Y_{1}(s) \\
& -\left[\sum_{i=1}^{n} Y^{i}(s) A_{x x}^{i}(s, X(s))+\sum_{j=1}^{m} \sum_{i=1}^{n} u^{j}(s) Y^{i}(s) B_{x x}^{i j}(s, X(s))+Q_{x x}(s, X(s))\right. \\
& \left.+\sum_{j=1}^{m} u^{j}(s) S_{x x}^{j}(s, X(s))+\frac{1}{2} \sum_{j, k=1}^{m} u^{j}(s) u^{k}(s) R_{x x}^{j k}(s, X(s))\right] X_{1}(s)
\end{aligned}
$$




$$
\begin{aligned}
& -\left[S_{x}(s, X(s))+\sum_{i=1}^{n} Y^{i}(s) \widetilde{B}_{x}^{i}(s, X(s))+\sum_{j=1}^{m} u^{j}(s) R_{x}^{j}(s, X(s))\right]^{T} v(s) \\
= & -\mathbf{A}(s)^{T} Y_{1}(s)-\mathbf{A}_{1}(s) X_{1}(s)-\mathbf{C}(s)^{T} v(s),
\end{aligned}
$$

where $\mathbf{A}(\cdot), \mathbf{A}_{1}(\cdot)$, and $\mathbf{C}(\cdot)$ are given by (5.5). Thus, $\left(X_{1}(\cdot), Y_{1}(\cdot)\right)$ solves (5.4).

Note that for given $(t, x) \in[0, T) \times \mathbb{R}^{n}$ and $u(\cdot) \in \mathcal{U}[t, T]$, both $X_{1}(\cdot)$ and $Y_{1}(\cdot)$ depend on $v(\cdot)$. It will be desirable to have a representation of $\left[D_{u u} J(t, x ; u(\cdot)) v(\cdot)\right]$ explicitly in terms of $v(\cdot)$. The following is such a result.

Theorem 5.2. Let (H1)-(H3) hold. For any $(t, x) \in[0, T) \times \mathbb{R}^{n}$ and $u(\cdot) \in \mathcal{U}[t, T]$, let $\mathbf{A}(\cdot)$, $\mathbf{A}_{1}(\cdot)$, and $\mathbf{C}(\cdot)$ be defined by (5.5). Then

$$
\left[D_{u u} J(t, x ; u(\cdot)) v(\cdot)\right](s)=R(s, X(s)) v(s)+\int_{t}^{T} \mathbf{F}(s, r) v(r) \mathrm{d} s, \quad \forall v(\cdot) \in \mathcal{U}[t, T]
$$

where

$$
\begin{aligned}
\mathbf{F}(s, r)= & B(s, X(s))^{T} \Phi_{\mathbf{A}}(T, s)^{T} G_{x x}(X(T)) \Phi_{\mathbf{A}}(T, r) B(r, X(r)) \\
& +\int_{s \vee r}^{T} B(s, X(s))^{T} \Phi_{\mathbf{A}}\left(r^{\prime}, s\right)^{T} \mathbf{A}_{1}\left(r^{\prime}\right) \Phi_{\mathbf{A}}\left(r^{\prime}, r\right) B(r, X(r)) \mathrm{d} r^{\prime} \\
& +\mathbf{C}(s) \Phi_{\mathbf{A}}(s, r) B(r, X(r)) I_{[t, s]}(r)+B(s, X(s))^{T} \Phi_{\mathbf{A}}(r, s)^{T} \mathbf{C}(r)^{T} I_{[s, T]}(r),
\end{aligned}
$$

and $\Phi_{\mathbf{A}}(\cdot, \cdot)$ is the fundamental matrix of $\mathbf{A}(\cdot)$, i.e., for any $\tau \in[t, T)$,

$$
\left\{\begin{array}{l}
\frac{\mathrm{d}}{\mathrm{d} s} \Phi_{\mathbf{A}}(s, \tau)=\mathbf{A}(s) \Phi_{\mathbf{A}}(s, \tau), \quad s \in[\tau, T] \\
\Phi_{\mathbf{A}}(\tau, \tau)=I
\end{array}\right.
$$

Proof. Let $\Phi_{\mathbf{A}}(\cdot, \cdot)$ be the fundamental matrix of $\mathbf{A}(\cdot)$. Then

$$
X_{1}(s)=\int_{t}^{s} \Phi_{\mathbf{A}}(s, r) B(r, X(r)) v(r) \mathrm{d} r, \quad s \in[t, T],
$$

and

$$
\begin{aligned}
Y_{1}(s)= & \Phi_{\mathbf{A}}(T, s)^{T} G_{x x}(X(T)) X_{1}(T)+\int_{s}^{T} \Phi_{\mathbf{A}}(r, s)^{T}\left[\mathbf{A}_{1}(r) X_{1}(r)+\mathbf{C}(r)^{T} v(r)\right] \mathrm{d} r \\
= & \Phi_{\mathbf{A}}(T, s)^{T} G_{x x}(X(T)) \int_{t}^{T} \Phi_{\mathbf{A}}(T, r) B(r, X(r)) v(r) \mathrm{d} r \\
& +\int_{s}^{T} \Phi_{\mathbf{A}}(r, s)^{T} \mathbf{A}_{1}(r) \int_{t}^{r} \Phi_{\mathbf{A}}\left(r, r^{\prime}\right) B\left(r^{\prime}, X\left(r^{\prime}\right)\right) v\left(r^{\prime}\right) \mathrm{d} r^{\prime} \mathrm{d} r+\int_{s}^{T} \Phi_{\mathbf{A}}(r, s)^{T} \mathbf{C}(r)^{T} v(r) \mathrm{d} r \\
= & \Phi_{\mathbf{A}}(T, s)^{T} G_{x x}(X(T)) \int_{t}^{T} \Phi_{\mathbf{A}}(T, r) B(r, X(r)) v(r) \mathrm{d} r \\
& +\int_{t}^{T}\left[\int_{s \vee r}^{T} \Phi_{\mathbf{A}}\left(r^{\prime}, s\right)^{T} \mathbf{A}_{1}\left(r^{\prime}\right) \Phi_{\mathbf{A}}\left(r^{\prime}, r\right) \mathrm{d} r^{\prime}\right] B(r, X(r)) v(r) \mathrm{d} r+\int_{s}^{T} \Phi_{\mathbf{A}}(r, s)^{T} \mathbf{C}(r)^{T} v(r) \mathrm{d} r
\end{aligned}
$$


Hence,

$$
\begin{aligned}
{[} & \left.D_{u u} J(t, x ; u(\cdot)) v(\cdot)\right](s)=R(s, X(s)) v(s)+B(s, X(s))^{T} Y_{1}(s)+\mathbf{C}(s) X_{1}(s) \\
= & R(s, X(s)) v(s)+\mathbf{C}(s) \int_{t}^{s} \Phi_{\mathbf{A}}(s, r) B(r, X(r)) v(r) \mathrm{d} r \\
& +B(s, X(s))^{T}\left[\Phi_{\mathbf{A}}(T, s)^{T} G_{x x}(X(T)) \int_{t}^{T} \Phi_{\mathbf{A}}(T, r) B(r, X(r)) v(r) \mathrm{d} r\right. \\
& \left.+\int_{t}^{T}\left(\int_{s \vee r}^{T} \Phi_{\mathbf{A}}\left(r^{\prime}, s\right)^{T} \mathbf{A}_{1}\left(r^{\prime}\right) \Phi_{\mathbf{A}}\left(r^{\prime}, r\right) \mathrm{d} r^{\prime}\right) B(r, X(r)) v(r) \mathrm{d} r+\int_{s}^{T} \Phi_{\mathbf{A}}(r, s)^{T} \mathbf{C}(r)^{T} v(r) \mathrm{d} r\right] \\
= & R(s, X(s)) v(s)+\int_{t}^{T} \mathbf{F}(s, r) v(r) \mathrm{d} s,
\end{aligned}
$$

proving (5.12).

We note that $\mathbf{F}(s, r)$ is depending on the given $u(\cdot)$ and is independent of $v(\cdot)$.

\section{InVERTIBILITY OF $D_{u u} J(t, x ; u(\cdot))$}

Having calculated $D_{u u} J(t, x ; u(\cdot))$, we now would like to look at conditions under which (4.8) holds. The following is a general result whose proof is straightforward.

Theorem 6.1. Theorem 6.1. Let (H1)-(H3) hold and let $(t, x) \in[0, T) \times \mathbb{R}^{n}, u(\cdot) \in \mathcal{U}[t, T]$ be given. Define $\mathbf{F}(\cdot, \cdot)$ by (5.13), with $(X(\cdot), Y(\cdot))$ being the solution to $(5.2)$. Then $D_{u u} J(t, x ; u(\cdot))$ admits a bounded inverse operator if and only if for any $w(\cdot) \in \mathcal{U}[t, T]$, the following second kind Fredholm integral equation is well-posed:

$$
w(s)=R(s, X(s)) v(s)+\int_{t}^{T} \mathbf{F}(s, r) v(r) \mathrm{d} r, \quad s \in[t, T] .
$$

A sufficient condition for the above is

$$
\left|R(s, X(s))^{-1} \mathbf{F}(s, r)\right| \leq \alpha<\frac{1}{T}, \quad s, r \in[0, T] .
$$

Practically, to use the above result, we need to first solve a (decoupled) two-point boundary value problem (5.2) to get $(X(\cdot), Y(\cdot))$. Then calculate $\mathbf{A}(\cdot), \mathbf{A}_{1}(\cdot)$ and $\mathbf{C}(\cdot)$, etc., followed by $\Phi_{\mathbf{A}}(\cdot, \cdot)$. Next, construct $\mathbf{F}(\cdot, \cdot)$ and then check see if the Fredholm integral equation (6.1) is well-posed or sufficiently look at if (6.2) holds. Roughly speaking, when $R(s, x)$ is sufficiently positive definite, $(6.2)$ will be true. Thus, we have some sufficient condition under which (4.8) holds. Let us look at a special case:

$$
B(t, x)=B(t), \quad R(t, x)=R(t), \quad S(t, x)=0, \quad(t, x) \in[0, T] \times \mathbb{R}^{n} .
$$

In this case,

$$
\left\{\begin{array}{l}
\mathbf{A}(s)=A_{x}(s, X(s)), \quad \mathbf{C}(s)=0, \\
\mathbf{A}_{1}(s)=\sum_{i=1}^{n} Y^{i}(s) A_{x x}^{i}(s, X(s))+Q_{x x}(s, X(s)), \quad s \in[0, T],
\end{array}\right.
$$

and $\mathbf{F}(\cdot, \cdot)$ has a relatively simpler form:

$$
\begin{aligned}
\mathbf{F}(s, r)= & B(s)^{T} \Phi_{\mathbf{A}}(T, s)^{T} G_{x x}(X(T)) \Phi_{\mathbf{A}}(T, r) B(r) \\
& +\int_{s \vee r}^{T} B(s)^{T} \Phi_{\mathbf{A}}\left(r^{\prime}, s\right)^{T} \mathbf{A}_{1}\left(r^{\prime}\right) \Phi_{\mathbf{A}}\left(r^{\prime}, r\right) B(r) \mathrm{d} r^{\prime}, \quad s, r \in[0, T] .
\end{aligned}
$$


We now look at some more direct sufficient conditions under which (4.8) holds. To this end, we first present the following proposition.

Theorem 6.2. Let $(\mathrm{H} 1)-(\mathrm{H} 3)$ hold. Let $(t, x) \in[0, T) \times \mathbb{R}^{n}$, and $u(\cdot) \in \mathcal{U}[t, T]$ be given. Let $(X(\cdot), Y(\cdot))$ be the solution to (5.2) and $\mathbf{A}(\cdot), \mathbf{A}_{1}(\cdot)$, and $\mathbf{C}(\cdot)$ be defined by (5.5). Then

$$
\begin{aligned}
\int_{t}^{T} & \left\langle\left[D_{u u} J(t, x ; u(\cdot)) v(\cdot)\right](s), v(s)\right\rangle \mathrm{d} s=\int_{t}^{T}\langle R(s, X(s)) v(s), v(s)\rangle \mathrm{d} s \\
& +\left\langle G_{x x}(X(T)) \int_{t}^{T} \Phi_{\mathbf{A}}(T, r) B(r, X(r)) v(r) \mathrm{d} r, \int_{t}^{T} \Phi_{\mathbf{A}}(T, r) B(r, X(r)) v(r) \mathrm{d} r\right\rangle \\
& +\int_{t}^{T}\left\langle\mathbf{A}_{1}(s) \int_{t}^{s} \Phi_{\mathbf{A}}(s, r) B(r, X(r)) v(r) \mathrm{d} r, \int_{t}^{s} \Phi_{\mathbf{A}}(s, r) B(r, X(r)) v(r) \mathrm{d} r\right\rangle \mathrm{d} s \\
& +2 \int_{t}^{T}\left\langle\mathbf{C}(s) \int_{t}^{s} \Phi_{\mathbf{A}}(s, r) B(r, X(r)) v(r) \mathrm{d} r, v(s)\right\rangle \mathrm{d} s .
\end{aligned}
$$

Further, suppose $\bar{G} \in \mathbb{S}_{+}^{n}$ and $\bar{Q}:[0, T] \rightarrow \mathbb{S}_{+}^{n}$ such that for some $\alpha \in(0,1)$,

$$
\left\{\begin{array}{l}
G_{x x}(X(T))+\bar{G} \geq 0, \\
\mathbf{A}_{1}(s)+\bar{Q}(s)-\alpha^{-1} \mathbf{C}(s)^{T} R(s, X(s))^{-1} \mathbf{C}(s) \geq 0, \quad s \in[t, T],
\end{array}\right.
$$

and

$$
(1-\alpha) R(s, X(s))-[\widehat{G}(t)+\widehat{Q}(s, t)] I \geq \delta I, \quad s \in[t, T],
$$

for some $\delta>0$, with

$$
\left\{\begin{array}{l}
\widehat{G}(t)=\left[\int_{t}^{T} \int_{t}^{T}\left|B(s, X(s))^{T} \Phi_{\mathbf{A}}(T, s)^{T} \bar{G} \Phi_{\mathbf{A}}(T, r) B(r, X(r))\right|^{2} \mathrm{~d} r \mathrm{~d} s\right]^{\frac{1}{2}} \\
\widehat{Q}(s, t)=\int_{s}^{T}\left[\int_{t}^{\tau} \int_{t}^{\tau}\left|B(r, X(r))^{T} \Phi_{\mathbf{A}}(\tau, r)^{T} \bar{Q}(\tau) \Phi_{\mathbf{A}}\left(\tau, r^{\prime}\right) B\left(r^{\prime}, X\left(r^{\prime}\right)\right)\right|^{2} \mathrm{~d} r^{\prime} \mathrm{d} r\right]^{\frac{1}{2}} \mathrm{~d} \tau
\end{array}\right.
$$

then

$$
D_{u u} J(t, x ; u(\cdot)) \geq \delta I .
$$

Proof. Let $(t, x) \in[0, T) \times \mathbb{R}^{n}$ and $u(\cdot) \in \mathcal{U}[t, T]$ be given. We have

$$
\begin{aligned}
& \int_{t}^{T} \int_{t}^{T}\langle\mathbf{F}(s, r) v(r), v(s)\rangle \mathrm{d} r \mathrm{~d} s \\
& =\left\langle G_{x x}(X(T)) \int_{t}^{T} \Phi_{\mathbf{A}}(T, r) B(r, X(r)) v(r) \mathrm{d} r, \int_{t}^{T} \Phi_{\mathbf{A}}(T, r) B(r, X(r)) v(r) \mathrm{d} r\right\rangle \\
& \quad+\int_{t}^{T}\left\langle\mathbf{A}_{1}(s) \int_{t}^{s} \Phi_{\mathbf{A}}(s, r) B(r, X(r)) v(r) \mathrm{d} r, \int_{t}^{s} \Phi_{\mathbf{A}}(s, r) B(r, X(r)) v(r) \mathrm{d} r\right\rangle \mathrm{d} s \\
& +\int_{t}^{T} \int_{t}^{s}\left\langle\mathbf{C}(s) \Phi_{\mathbf{A}}(s, r) B(r, X(r)) v(r), v(s)\right\rangle \mathrm{d} r \mathrm{~d} s \\
& +\int_{t}^{T} \int_{s}^{T}\left\langle B(s, X(s))^{T} \Phi_{\mathbf{A}}(r, s)^{T} \mathbf{C}(r)^{T} v(r), v(s)\right\rangle \mathrm{d} r \mathrm{~d} s
\end{aligned}
$$




$$
\begin{aligned}
= & \left\langle G_{x x}(X(T)) \int_{t}^{T} \Phi_{\mathbf{A}}(T, r) B(r, X(r)) v(r) \mathrm{d} r, \int_{t}^{T} \Phi_{\mathbf{A}}(T, r) B(r, X(r)) v(r) \mathrm{d} r\right\rangle \\
& +\int_{t}^{T}\left\langle\mathbf{A}_{1}(s) \int_{t}^{s} \Phi_{\mathbf{A}}(s, r) B(r, X(r)) v(r) \mathrm{d} r, \int_{t}^{s} \Phi_{\mathbf{A}}(s, r) B(r, X(r)) v(r) \mathrm{d} r\right\rangle \mathrm{d} s \\
& +2 \int_{t}^{T}\left\langle\mathbf{C}(s) \int_{t}^{s} \Phi_{\mathbf{A}}(s, r) B(r, X(r)) v(r) \mathrm{d} r, v(s)\right\rangle \mathrm{d} s .
\end{aligned}
$$

This proves (6.6). From this, one further has

$$
\begin{aligned}
& \int_{t}^{T}\left\langle\left[D_{u u} J(t, x ; u(\cdot)) v(\cdot)\right](s), v(s)\right\rangle \mathrm{d} s=\int_{t}^{T}\langle R(s, X(s)) v(s), v(s)\rangle \mathrm{d} s \\
& +\left\langle G_{x x}(X(T)) \int_{t}^{T} \Phi_{\mathbf{A}}(T, r) B(r, X(r)) v(r) \mathrm{d} r, \int_{t}^{T} \Phi_{\mathbf{A}}(T, r) B(r, X(r)) v(r) \mathrm{d} r\right\rangle \\
& +\int_{t}^{T}\left\langle\mathbf{A}_{1}(s) \int_{t}^{s} \Phi_{\mathbf{A}}(s, r) B(r, X(r)) v(r) \mathrm{d} r, \int_{t}^{s} \Phi_{\mathbf{A}}(s, r) B(r, X(r)) v(r) \mathrm{d} r\right\rangle \mathrm{d} s \\
& +2 \int_{t}^{T}\left\langle\mathbf{C}(s) \int_{t}^{s} \Phi_{\mathbf{A}}(s, r) B(r, X(r)) v(r) \mathrm{d} r, v(s)\right\rangle \mathrm{d} s \\
& =\int_{t}^{T}\langle(1-\alpha) R(s, X(s)) v(s), v(s)\rangle \mathrm{d} s+\int_{t}^{T}\left(\left|\alpha^{\frac{1}{2}} R(s, X(s))^{\frac{1}{2}} v(s)\right|^{2} \mathrm{~d} s\right. \\
& +2\left\langle\alpha^{-\frac{1}{2}} R(s, X(s))^{-\frac{1}{2}} \mathbf{C}(s) \int_{t}^{s} \Phi_{\mathbf{A}}(s, r) B(r, X(r)) v(r) \mathrm{d} r, \alpha^{\frac{1}{2}} R(s, X(s))^{\frac{1}{2}} v(s)\right\rangle \\
& \left.+\left|\alpha^{-\frac{1}{2}} R(s, X(s))^{-\frac{1}{2}} \mathbf{C}(s) \int_{t}^{s} \Phi_{\mathbf{A}}(s, r) B(r, X(r)) v(r) \mathrm{d} r\right|^{2}\right) \mathrm{d} s \\
& +\left\langle G_{x x}(X(T)) \int_{t}^{T} \Phi_{\mathbf{A}}(T, r) B(r, X(r)) v(r) \mathrm{d} r, \int_{t}^{T} \Phi_{\mathbf{A}}(T, r) B(r, X(r)) v(r) \mathrm{d} r\right\rangle \\
& +\int_{t}^{T}\left\langle\left[\mathbf{A}_{1}(s)-\alpha^{-1} \mathbf{C}(s)^{T} R(s, X(s))^{-1} \mathbf{C}(s)\right] \int_{t}^{s} \Phi_{A}(s, r) B(r, X(r)) v(r) \mathrm{d} r,\right. \\
& \left.\int_{t}^{s} \Phi_{A}(s, r) B(r, X(r)) v(r) \mathrm{d} r\right\rangle \mathrm{d} s \\
& =\int_{t}^{T}\langle(1-\alpha) R(s, X(s)) v(s), v(s)\rangle \mathrm{d} s \\
& +\int_{t}^{T}\left|\alpha^{\frac{1}{2}} R(s, X(s))^{\frac{1}{2}} v(s)+\alpha^{-\frac{1}{2}} R(s, X(s))^{-\frac{1}{2}} \mathbf{C}(s) \int_{t}^{s} \Phi_{\mathbf{A}}(s, r) B(r, X(r)) v(r) \mathrm{d} r\right|^{2} \mathrm{~d} s \\
& +\left\langle\left[G_{x x}(X(T))+\bar{G}\right] \int_{t}^{T} \Phi_{\mathbf{A}}(T, r) B(r, X(r)) v(r) \mathrm{d} r, \int_{t}^{T} \Phi_{\mathbf{A}}(T, r) B(r, X(r)) v(r) \mathrm{d} r\right\rangle \\
& +\int_{t}^{T}\left\langle\left[\mathbf{A}_{1}(s)+\bar{Q}(s)-\alpha^{-1} \mathbf{C}(s)^{T} R(s, X(s))^{-1} \mathbf{C}(s)\right] \int_{t}^{s} \Phi_{A}(s, r) B(r, X(r)) v(r) \mathrm{d} r\right. \\
& \left.\int_{t}^{s} \Phi_{A}(s, r) B(r, X(r)) v(r) \mathrm{d} r\right\rangle \mathrm{d} s
\end{aligned}
$$




$$
\begin{aligned}
& -\left\langle\bar{G} \int_{t}^{T} \Phi_{\mathbf{A}}(T, r) B(r, X(r)) v(r) \mathrm{d} r, \int_{t}^{T} \Phi_{\mathbf{A}}(T, r) B(r, X(r)) v(r) \mathrm{d} r\right\rangle \\
& -\int_{t}^{T}\left\langle\bar{Q}(s) \int_{t}^{s} \Phi_{\mathbf{A}}(s, r) B(r) v(r) \mathrm{d} r, \int_{t}^{s} \Phi_{\mathbf{A}}(s, r) B(r) v(r) \mathrm{d} r\right\rangle \mathrm{d} s \\
\geq & \int_{t}^{T}\langle(1-\alpha) R(s, X(s)) v(s), v(s)\rangle \mathrm{d} s-\left\langle\bar{G} \int_{t}^{T} \Phi_{\mathbf{A}}(T, r) B(r, X(r)) v(r) \mathrm{d} r, \int_{t}^{T} \Phi_{\mathbf{A}}(T, r) B(r, X(r)) v(r) \mathrm{d} r\right\rangle \\
& -\int_{t}^{T}\left\langle\bar{Q}(s) \int_{t}^{s} \Phi_{\mathbf{A}}(s, r) B(r) v(r) \mathrm{d} r, \int_{t}^{s} \Phi_{\mathbf{A}}(s, r) B(r) v(r) \mathrm{d} r\right\rangle \mathrm{d} s .
\end{aligned}
$$

Note that

$$
\begin{aligned}
& \left\langle\bar{G} \int_{t}^{T} \Phi_{\mathbf{A}}(T, r) B(r, X(r)) v(r) \mathrm{d} r, \int_{t}^{T} \Phi_{\mathbf{A}}(T, r) B(r, X(r)) v(r) \mathrm{d} r\right\rangle \\
& =\int_{t}^{T}\left\langle\int_{t}^{T} B(s, X(s))^{T} \Phi_{\mathbf{A}}(T, s)^{T} \bar{G} \Phi_{\mathbf{A}}(T, r) B(r, X(r)) v(r) \mathrm{d} r, v(s)\right\rangle \mathrm{d} s \\
& \leq\left(\int_{t}^{T}\left|\int_{t}^{T} B(s, X(s))^{T} \Phi_{\mathbf{A}}(T, s)^{T} \bar{G} \Phi_{\mathbf{A}}(T, r) B(r, X(r)) v(r) \mathrm{d} r\right|^{2} \mathrm{~d} s\right)^{\frac{1}{2}}\left(\int_{t}^{T}|v(s)|^{2} \mathrm{~d} s\right)^{\frac{1}{2}} \\
& \leq\left[\int_{t}^{T}\left(\int_{t}^{T}\left|B(s, X(s))^{T} \Phi_{\mathbf{A}}(T, s)^{T} \bar{G} \Phi_{\mathbf{A}}(T, r) B(r, X(r))\right|^{2} \mathrm{~d} r \mathrm{~d} s\right)\left(\int_{t}^{T}|v(r)|^{2} \mathrm{~d} r\right)\right]^{\frac{1}{2}}\left(\int_{t}^{T}|v(r)|^{2} \mathrm{~d} r\right)^{\frac{1}{2}} \\
& =\left[\int_{t}^{T} \int_{t}^{T}\left|B(s, X(s))^{T} \Phi_{\mathbf{A}}(T, s)^{T} \bar{G} \Phi_{\mathbf{A}}(T, r) B(r, X(r))\right|^{2} \mathrm{~d} r \mathrm{~d} s\right]^{\frac{1}{2}} \int_{t}^{T}|v(r)|^{2} \mathrm{~d} r \equiv \int_{t}^{T}\langle\widehat{G}(t) v(s), v(s)\rangle \mathrm{d} s,
\end{aligned}
$$

and similarly,

$$
\begin{aligned}
& \left.\int_{t}^{T}\left\langle\bar{Q}(s) \int_{t}^{s} \Phi_{\mathbf{A}}(s, r) B(r, X(r))\right) v(r) \mathrm{d} r, \int_{t}^{s} \Phi_{\mathbf{A}}(s, r) B(r, X(r)) v(r) \mathrm{d} r\right\rangle \mathrm{d} s \\
& =\int_{t}^{T} \int_{t}^{s}\left\langle\int_{t}^{s} B\left(r^{\prime}, X\left(r^{\prime}\right)\right)^{T} \Phi_{\mathbf{A}}\left(s, r^{\prime}\right) \bar{Q}(s) \Phi_{\mathbf{A}}(s, r) B(r, X(r)) v(r) \mathrm{d} r, v\left(r^{\prime}\right)\right\rangle \mathrm{d} r^{\prime} \mathrm{d} s \\
& \leq \int_{t}^{T}\left[\int_{t}^{s} \int_{t}^{s}\left|B(r, X(r))^{T} \Phi_{\mathbf{A}}(s, r)^{T} \bar{Q}(s) \Phi_{A}\left(s, r^{\prime}\right) B\left(r^{\prime}, X\left(r^{\prime}\right)\right)\right|^{2} \mathrm{~d} r^{\prime} \mathrm{d} r\right]_{t}^{\frac{1}{2}}|v(r)|^{2} \mathrm{~d} r \mathrm{~d} s \\
& =\int_{t}^{T}\left\{\int_{\tau}^{T}\left[\int_{t}^{s} \int_{t}^{s} \mid B(r, X(r))^{T} \Phi_{\mathbf{A}}(t, x)^{T} \bar{Q}(s) \Phi_{\mathbf{A}}\left(s, r^{\prime}\right) B\left(r^{\prime},\left.X\left(r^{\prime}\right)\right|^{2} \mathrm{~d} r^{\prime} \mathrm{d} r\right]^{\frac{1}{2}} \mathrm{~d} s\right\}|v(\tau)|^{2} \mathrm{~d} \tau\right. \\
& \equiv \int_{t}^{T} \widehat{Q}(s, t)|v(s)|^{2} \mathrm{~d} s,
\end{aligned}
$$


where $\widehat{G}(t)$ and $\widehat{Q}(s, t)$ are given by $(6.9)$. Consequently,

$$
\begin{aligned}
& \int_{t}^{T}\left\langle\left[D_{u u} J(t, x ; u(\cdot)) v(\cdot)\right](s), v(s)\right\rangle \mathrm{d} s \\
& \geq \int_{t}^{T}\langle(1-\alpha) R(s, X(s)) v(s), v(s)\rangle \mathrm{d} s-\left\langle\bar{G} \int_{t}^{T} \Phi_{\mathbf{A}}(T, r) B(r, X(r)) v(r) \mathrm{d} r, \int_{t}^{T} \Phi_{\mathbf{A}}(T, r) B(r, X(r)) v(r) \mathrm{d} r\right\rangle \\
& \quad-\int_{t}^{T}\left\langle\bar{Q}(s) \int_{t}^{s} \Phi_{\mathbf{A}}(s, r) B(r, X(r)) v(r) \mathrm{d} r, \int_{t}^{s} \Phi_{\mathbf{A}}(s, r) B(r, X(r)) v(r) \mathrm{d} r\right\rangle \mathrm{d} s \\
& \geq \int_{t}^{T}\langle((1-\alpha) R(s, X(s))-[\widehat{G}(t)+\widehat{Q}(s, t)] I) v(s), v(s)\rangle \mathrm{d} s .
\end{aligned}
$$

Hence, (6.10) follows.

We now look at some interesting cases.

\subsection{Linear quadratic case}

Let

$$
\left\{\begin{array}{l}
A(t, x)=A(t) x, \quad B(t, x)=B(t), \quad Q(t, x)=\frac{1}{2}\langle Q(t) x, x\rangle, \\
S(t, s)=S(t) x, \quad R(t, x)=R(t), \quad G(x)=\frac{1}{2}\langle G x, x\rangle .
\end{array}\right.
$$

This is a classical LQ case. In this case,

$$
\mathbf{A}(s)=A(s), \quad \mathbf{A}_{1}(s)=Q(s), \quad \mathbf{C}(s)=S(s), \quad s \in[0, T] .
$$

Then (6.7) holds if

$$
\left\{\begin{array}{l}
G+\bar{G} \geq 0, \\
Q(s)+\bar{Q}(s)-\alpha^{-1} S(s)^{T} R(s)^{-1} S(s) \geq 0, \quad s \in[0, T] .
\end{array}\right.
$$

for some $\bar{G} \in \mathbb{S}_{+}^{n}, \bar{Q}:[0, T] \rightarrow \mathbb{S}_{+}^{n}$, and $\alpha \in(0,1)$. In this case, $\Phi_{A}(\cdot, \cdot)$, the fundamental matrix of $A(\cdot)$, is independent of $u(\cdot), X(\cdot)$ and $Y(\cdot)$. Consequently,

$$
\left\{\begin{array}{l}
\widehat{G}(t)=\left[\int_{t}^{T} \int_{t}^{T}\left|B(s)^{T} \Phi_{A}(T, s)^{T} \bar{G} \Phi_{A}(T, r) B(r)\right|^{2} \mathrm{~d} r \mathrm{~d} s\right]^{\frac{1}{2}}, \\
\widehat{Q}(s, t)=\int_{s}^{T}\left[\int_{t}^{\tau} \int_{t}^{\tau}\left|B(r)^{T} \Phi_{A}(\tau, r)^{T} \bar{Q}(\tau) \Phi_{A}\left(\tau, r^{\prime}\right) B\left(r^{\prime}\right)\right|^{2} \mathrm{~d} r^{\prime} \mathrm{d} r\right]^{\frac{1}{2}} \mathrm{~d} \tau .
\end{array}\right.
$$

are independent of $u(\cdot), X(\cdot)$, and $Y(\cdot)$. Then, by Theorem 6.2 , we obtain the following result.

Proposition 6.3. Let (H1)-(H3) and (6.11) hold. Suppose there exist $\alpha \in(0,1)$, and $\bar{G} \in \mathbb{S}_{+}^{n}, \bar{Q}:[t, T] \rightarrow \mathbb{S}_{+}^{n}$ such that

$$
Q(s)+\bar{Q}(s)-\alpha^{-1} S(s)^{T} R(s)^{-1} S(s) \geq 0, \quad G+\bar{G} \geq 0, \quad s \in[t, T],
$$

and

$$
(1-\alpha) R(s)-[\widehat{G}(t)+\widehat{Q}(s, t)] I \geq \delta I, \quad s \in[t, T],
$$

for some $\delta>0$, where $\widehat{G}(t)$ and $\widehat{Q}(s, t)$ are defined by $(6.12)$. Then

$$
D_{u u} J(t, x ; u(\cdot)) \geq \delta I, \quad \forall u(\cdot) \in \mathcal{U}[t, T] .
$$


We point out that under the following classical conditions for LQ problems:

$$
R(s) \geq \delta I, \quad Q(s)-S(s)^{T} R(s)^{-1} S(s) \geq 0, \quad G \geq 0,
$$

we need only take

$$
\alpha=0, \quad \bar{G}=0, \quad \bar{Q}(\cdot)=0 .
$$

Therefore, the above result covers the classical LQ problem. Further, Proposition 6.3 shows that for LQ problems, the failure of the last two conditions in (6.15) can be compensated by the sufficient positive definiteness of $R(s)$. On the other hand, we see that due to the nature of LQ problem, the positive definiteness of $D_{u u} J(t, x ; u(\cdot))$ obtained above is automatically uniform in $u(\cdot)$.

\subsection{Linear semi-convex case}

Let us first assume the following:

$$
\left\{\begin{array}{l}
A(t, x)=A(t) x, \quad B(t, x)=B(t), \quad S(t, x)=0, \quad R(t, x)=R(t) \geq \delta I, \\
x \mapsto Q(t, x), \quad x \mapsto G(x) \quad \text { are convex. }
\end{array}\right.
$$

In the above case, we have a linear state equation and a convex cost functional. This is a natural generalization of LQ case and we refer to it as linear-convex problem. Such kind of problems were carefully studied in $[22,23]$ by means of the so-called quasi-Riccati equation.

Note that under (6.16), it is straightforward that $u(\cdot) \mapsto J(t, x ; u(\cdot))$ is uniformly convex. In our framework, one has

$$
\mathbf{A}(s)=A(s), \quad \mathbf{A}_{1}(s)=Q_{x x}(s, X(s)), \quad \mathbf{C}(s)=0, \quad s \in[0, T] .
$$

Then

$$
\begin{aligned}
& \int_{t}^{T}\left\langle\left[D_{u u} J(t, x ; u(\cdot)) v(\cdot)\right](s), v(s)\right\rangle \mathrm{d} s \\
& =\int_{t}^{T}\langle R(s) v(s), v(s)\rangle \mathrm{d} s+\left\langle G_{x x}(X(T)) \int_{t}^{T} \Phi_{A}(T, r) B(r) v(r) \mathrm{d} r, \int_{t}^{T} \Phi_{A}(T, r) B(r) v(r) \mathrm{d} r\right\rangle \\
& \quad+\int_{t}^{T}\left\langle Q_{x x}(s, X(s)) \int_{t}^{s} \Phi_{A}(s, r) B(r) v(r) \mathrm{d} r, \int_{t}^{s} \Phi_{A}(s, r) B(r) v(r) \mathrm{d} r\right\rangle \mathrm{d} s \\
& \geq \int_{t}^{T}\langle R(s) v(s), v(s)\rangle \mathrm{d} s \geq \delta \int_{t}^{T}|v(s)|^{2} \mathrm{~d} s,
\end{aligned}
$$

proving the uniform convexity of the map $u(\cdot) \mapsto J(t, x ; u(\cdot))$.

We can actually do a little bit more. Here is the result whose proof follows from Theorem 6.2 easily.

Proposition 6.4. Let (H1)-(H3) hold such that for some $\bar{Q}:[0, T] \rightarrow \mathbb{S}_{+}^{n}$ and $\bar{G} \in \mathbb{S}_{+}^{n}$,

$$
A(t, x)=A(t) x, \quad B(t, x)=B(t), \quad S(t, x)=S(t) x, \quad R(t, x)=R(t),
$$

and

$$
\left\{\begin{array}{l}
Q_{x x}(s, x)+\bar{Q}(s)-\alpha^{-1} S(s)^{T} R(s)^{-1} S(s) \geq 0, \quad(s, x) \in[t, T] \times \mathbb{R}^{n}, \\
G_{x x}(x)+\bar{G} \geq 0, \quad s \in[t, T]
\end{array}\right.
$$

and

$$
(1-\alpha) R(s)-[\widehat{G}(t)+\widehat{Q}(s, t)] I \geq \delta I, \quad s \in[t, T],
$$

for some $\delta>0$, where $\widehat{G}(t)$ and $\widehat{Q}(s, t)$ are defined by $(6.12)$. Then $D_{u u} J(t, x ; u(\cdot))$ is uniformly positive definite. 
Recall that maps $x \mapsto Q(t, x)$ and $x \mapsto G(x)$ as semi-convex maps if there is a constant $K>0$ such that

$$
x \mapsto Q(t, x)+K|x|^{2}, \quad x \mapsto Q(x)+K|x|^{2}
$$

are convex. It is clear that under (6.18), $x \mapsto Q(t, x)$ and $x \mapsto G(x)$ are semi-convex. Hence, the associated problem is referred to as a linear semi-convex problem. Our result basically shows that the possible deviation from the convexity of the maps $x \mapsto Q(t, x)$ and $x \mapsto G(x)$ could be possibly compensated by the sufficient positive definiteness of $R(\cdot)$.

\subsection{Another nonlinear case}

We now impose the following conditions:

$$
B(t, x)=B(t), \quad R(t, x)=R(t), \quad S(t, x)=0, \quad(t, x) \in[0, T] \times \mathbb{R}^{n} .
$$

Note that we still allow $x \mapsto(A(t, x), Q(t, x), G(x))$ to be nonlinear. In the current case, we have

$$
\left\{\begin{array}{l}
\mathbf{A}(s)=A_{x}(s, X(s)), \quad \mathbf{C}(s)=0, \\
\mathbf{A}_{1}(s)=\sum_{i=1}^{n} Y^{i}(s) A_{x x}^{i}(s, X(s))+Q_{x x}(s, X(s)) .
\end{array}\right.
$$

Also,

$$
\begin{aligned}
& \int_{t}^{T}\left\langle\left[D_{u u} J(t, x ; u(\cdot)) v(\cdot)\right](s), v(s)\right\rangle \mathrm{d} s \\
& \geq \int_{t}^{T}\langle(1-\alpha) R(s) v(s), v(s)\rangle \mathrm{d} s-\left\langle\bar{G} \int_{t}^{T} \Phi_{\mathbf{A}}(T, r) B(r) v(r) \mathrm{d} r, \int_{t}^{T} \Phi_{\mathbf{A}}(T, r) B(r) v(r) \mathrm{d} r\right\rangle \\
& \quad-\int_{t}^{T}\left\langle\bar{Q}(s) \int_{t}^{s} \Phi_{\mathbf{A}}(s, r) B(r) v(r) \mathrm{d} r \int_{t}^{s} \Phi_{\mathbf{A}}(s, r) B(r) v(r) \mathrm{d} r\right\rangle \mathrm{d} s \\
& \quad+\left\langle\left(G_{x x}(X(T))+\bar{G}\right) \int_{t}^{T} \Phi_{\mathbf{A}}(T, r) B(r) v(r) \mathrm{d} r, \int_{t}^{T} \Phi_{\mathbf{A}}(T, r) B(r) v(r) \mathrm{d} r\right\rangle \\
& \quad+\int_{t}^{T}\left\langle\left[\mathbf{A}_{1}(s)+\bar{Q}(s)\right] \int_{t}^{s} \Phi_{\mathbf{A}}(s, r) B(r) v(r) \mathrm{d} r, \int_{t}^{s} \Phi_{\mathbf{A}}(s, r) B(r) v(r) \mathrm{d} r\right\rangle \mathrm{d} s \\
& =\int_{t}^{T}\langle\{(1-\alpha) R(s)-[\widehat{G}(t)+\widehat{Q}(s, t)] I\} v(s), v(s)\rangle \mathrm{d} s+\mathbb{I}_{2}+\mathbb{I}_{3},
\end{aligned}
$$

where $\widehat{G}(\cdot)$ and $\widehat{Q}(\cdot)$ are defined by the following:

$$
\left\{\begin{array}{l}
\widehat{G}(t)=\left[\int_{t}^{T} \int_{t}^{T}\left|B(s)^{T} \Phi_{\mathbf{A}}(T, s)^{T} \bar{G} \Phi_{\mathbf{A}}(T, r) B(r)\right|^{2} \mathrm{~d} r \mathrm{~d} s\right]^{\frac{1}{2}}, \\
\widehat{Q}(s, t)=\int_{s}^{T}\left[\int_{t}^{\tau} \int_{t}^{\tau}\left|B(r)^{T} \Phi_{\mathbf{A}}(\tau, r)^{T} \bar{Q}(\tau) \Phi_{\mathbf{A}}\left(\tau, r^{\prime}\right) B\left(r^{\prime}\right)\right|^{2} \mathrm{~d} r^{\prime} \mathrm{d} r\right]^{\frac{1}{2}} \mathrm{~d} \tau
\end{array}\right.
$$

and

$$
\left\{\begin{array}{l}
\mathbb{I}_{2}=\left\langle\left(G_{x x}(X(T))+\bar{G}\right) \int_{t}^{T} \Phi_{\mathbf{A}}(T, r) B(r) v(r) \mathrm{d} r, \int_{t}^{T} \Phi_{\mathbf{A}}(T, r) B(r) v(r) \mathrm{d} r\right\rangle \\
\mathbb{I}_{3}=\int_{t}^{T}\left\langle\left[\mathbf{A}_{1}(s)+\bar{Q}(s)\right] \int_{t}^{s} \Phi_{\mathbf{A}}(s, r) B(r) v(r) \mathrm{d} r, \int_{t}^{s} \Phi_{\mathbf{A}}(s, r) B(r) v(r) \mathrm{d} r\right\rangle \mathrm{d} s .
\end{array}\right.
$$


Naturally, we may still assume

$$
G_{x x}(x)+\bar{G} \geq 0, \quad \forall x \in \mathbb{R}^{n},
$$

which implies $\mathbb{I}_{2} \geq 0$. To ensure $\mathbb{I}_{3} \geq 0$, we may let

$$
\mathbf{A}_{1}(s)+\bar{Q}(s) \equiv \sum_{i=1}^{n} Y^{i}(s) A_{x x}^{i}(s, X(s))+Q_{x x}(s, X(s))+\bar{Q}(s) \geq 0 .
$$

Note that in the current case,

$$
\left\{\begin{array}{l}
\dot{X}(s)=A(s, X(s))+B(s) u(s) \\
\dot{Y}(s)=-A_{x}(s, X(s))^{T} Y(s)-Q_{x}(s, X(s))^{T} \\
X(t)=x, \quad Y(T)=G_{x}(X(T))^{T}
\end{array}\right.
$$

Thus, if

$$
\left|A_{x}(t, x)\right| \leq L_{A}, \quad\left|Q_{x}(t, x)\right| \leq L_{Q}, \quad\left|G_{x}(x)\right| \leq L_{G}, \quad(t, x) \in[0, T] \times \mathbb{R}^{n},
$$

for some constants $L_{A}, L_{Q}, L_{G}>0$, then

$$
|Y(s)| \leq L_{G}+\int_{s}^{T}\left(L_{A}|Y(r)|+L_{Q}\right) \mathrm{d} r, \quad s \in[t, T] .
$$

By Gronwall's inequality, we have

$$
|Y(s)| \leq \mathrm{e}^{L_{A}(T-s)} L_{G}+\frac{L_{Q}}{L_{A}}\left(\mathrm{e}^{L_{A}(T-s)}-1\right) \leq L_{Y}, \quad s \in[t, T] .
$$

with

$$
L_{Y}=L_{G} \mathrm{e}^{L_{A} T}+\frac{L_{Q}}{L_{A}}\left(\mathrm{e}^{L_{A} T}-1\right)
$$

Then we have the following result.

Proposition 6.5. Let $\bar{G} \in \mathbb{S}^{n}$ and $\bar{Q}:[0, T] \rightarrow \mathbb{S}^{n}$ such that for some $\alpha \in(0,1)$ and $\delta>0$,

$$
\begin{gathered}
G_{x x}(x)+\bar{G} \geq 0, \quad x \in \mathbb{R}^{n}, \\
R(s)-[\widehat{G}(t)+\widehat{Q}(s)] I \geq \delta I, \quad 0 \leq t \leq s \leq T,
\end{gathered}
$$

with $\widehat{G}(\cdot)$ and $\widehat{Q}(\cdot)$ defined as in $(6.22)$, and

$$
Q_{x x}(s, x)+\bar{Q}(s)-L_{Y} \sum_{i=1}^{n}\left|A_{x x}^{i}(s, x)\right| \geq 0, \quad(s, x) \in[0, T] \times \mathbb{R}^{n} .
$$

Then $D_{u u} J(t, x ; u(\cdot))$ is positive definite.

From (6.30), we see that due to the nonlinearity of $x \mapsto A(t, x)$, we basically need the semi-convexity of $x \mapsto Q(t, x)$ and the sufficient positive definiteness of $R(\cdot)$ to compensate.

Along the above line, it is possible to give many other similar conditions under which (4.8) holds. We prefer not to get into such kind of details. 


\section{QUASI-RICCATI EQUATION}

Let us assume the following:

$$
B(t, x)=B(t), \quad R(t, x)=R(t), \quad(t, x) \in[0, T] \times \mathbb{R}^{n} .
$$

We have seen from Theorem 4.2 that when $(\mathrm{H} 1)-(\mathrm{H} 3)$ and $(4.8)$ hold, the value function $V(\cdot, \cdot)$ is actually twice continuously differentiable. Consequently, $V(\cdot, \cdot)$ satisfies the HJB equation in the classical sense, and by the smoothness of the coefficients, we can differentiate the equation once. Note that in the current case, our HJB equation reads:

$$
\left\{\begin{array}{l}
V_{t}(t, x)+V_{x}(t, x) A(t, x)+Q(t, x) \\
\quad-\frac{1}{2}\left[V_{x}(t, x) B(t)+S(t, x)^{T}\right] R(t)^{-1}\left[B(t, x)^{T} V_{x}(t, x)^{T}+S(t, x)\right]=0, \quad(t, x) \in[0, T) \times \mathbb{R}^{n}, \\
V(T, x)=G(x), \quad x \in \mathbb{R}^{n} .
\end{array}\right.
$$

Now, we define

$$
P(t, x)=V_{x}(t, x)^{T}
$$

Then

$$
P_{x}(t, x)=V_{x x}(t, x)=P_{x}(t, x)^{T}, \quad \forall(t, x) \in[0, T) \times \mathbb{R}^{n},
$$

and the following holds:

$$
\left\{\begin{array}{l}
P_{t}(t, x)+P_{x}(t, x) A(t, x)+A_{x}(t, x)^{T} P(t, x)+Q_{x}(t, x)^{T} \\
\quad-\left[P_{x}(t, x) B(t)+S_{x}(t, x)^{T}\right] R(t)^{-1}\left[B(t)^{T} P(t, x)+S(t, x)\right]=0, \quad(t, x) \in[0, T] \times \mathbb{R}^{n}, \\
P(T, x)=G_{x}(x), \quad x \in \mathbb{R}^{n} .
\end{array}\right.
$$

The above is called a Quasi-Riccati equation of Problem (AQ). This is an extension of that presented in [23] for linear-convex problems. We now have the following result.

Theorem 7.1. Let (H1)-(H3) hold. Let $\left(X^{*}(\cdot), u^{*}(\cdot)\right)$ be an optimal pair of Problem (AQ). Suppose the value function $V(\cdot, \cdot)$ of Problem $(A Q)$ is twice differentiable. Then $P(\cdot, \cdot) \equiv V_{x}(\cdot, \cdot)^{T}$ is a solution to the quasi-Riccati equation (7.3), and the optimal control $u^{*}(\cdot)$ admits the following state feedback representation:

$$
u^{*}(s)=-R(s)^{-1}\left[B(s)^{T} P\left(s, X^{*}(s)\right)+S\left(x, X^{*}(s)\right)\right], \quad s \in[t, T] .
$$

Proof. It is known that if $\left(X^{*}(\cdot), u^{*}(\cdot)\right)$ is an optimal pair of Problem (AQ) for the initial pair $(t, x) \in[0, T) \times \mathbb{R}^{n}$, and $Y(\cdot)$ is the solution to the corresponding adjoint equation, then

$$
Y(s)=V_{x}\left(s \cdot X^{*}(s)\right)^{T}=P\left(s, X^{*}(s)\right), \quad s \in[t, T],
$$

and

$$
\begin{aligned}
u^{*}(s) & =-R(s)^{-1}\left[B(s)^{T} Y(s)+S\left(x, X^{*}(s)\right)\right] \\
& =-R(s)^{-1}\left[B(s)^{T} P\left(s, X^{*}(s)\right)+S\left(x, X^{*}(s)\right)\right], \quad s \in[t, T] .
\end{aligned}
$$

This proves our conclusion.

In the case

$$
A(t, x)=A(t) x, \quad Q(t, x)=\frac{1}{2}\langle Q(t) x, x\rangle, \quad S(t, x)=S(t) x, \quad G(x)=\frac{1}{2}\langle G x, x\rangle
$$


we see that

$$
P(t, x)=P(t) x, \quad(t, x) \in[0, T] \times \mathbb{R}^{n},
$$

with $P(\cdot)$ being the solution to the following:

$$
\left\{\begin{array}{l}
\dot{P}(t)+P(t) A(t)+A(t)^{T} P(t)+Q(t)-\left[P(t) B(t)+S(t)^{T}\right] R(t)^{-1}\left[B(t)^{T} P(t)+S(t)\right]=0, \quad t \in[0, T], \\
P(T)=G,
\end{array}\right.
$$

which is the Riccati equation for a standard LQ problem.

To conclude this section, we present two illustrative examples.

Example 7.2. Consider the following one-dimensional linear controlled system:

$$
\left\{\begin{array}{l}
\dot{X}(s)=X(s)+u(s), \quad s \in[t, T] \\
X(t)=x
\end{array}\right.
$$

with cost functional:

$$
J(t, x ; u(\cdot))=\int_{t}^{T}\left(-\frac{1}{2} \cos ^{2} X(s)+\frac{1}{2} \rho u(s)^{2}\right) \mathrm{d} s-\frac{1}{2} \sin ^{2} X(T),
$$

where $\rho>0$. This is a linear-semi-convex problem. According to Proposition 6.4, we may choose

$$
\bar{Q}(t) \equiv \bar{G}=1 .
$$

Then

$$
\widehat{G}(t)=\int_{t}^{T} \mathrm{e}^{2(T-s)} \mathrm{d} s=\frac{\mathrm{e}^{2(T-t)}-1}{2} \leq \widehat{G}(0)=\frac{\mathrm{e}^{2 T}-1}{2},
$$

and

$$
\begin{aligned}
\widehat{Q}(s) & =\int_{s}^{T} \int_{s}^{\tau} \mathrm{e}^{2(\tau-r)} \mathrm{d} r \mathrm{~d} \tau=\frac{1}{2} \int_{s}^{T}\left(\mathrm{e}^{2(\tau-s)}-1\right) \mathrm{d} s \\
& =\frac{\mathrm{e}^{2(T-s)}-1}{4}-\frac{T-s}{2} \leq \widehat{Q}(0)=\frac{\mathrm{e}^{2 T}-1}{4}-\frac{T}{2} .
\end{aligned}
$$

Hence, under condition

$$
\rho>\frac{\mathrm{e}^{2 T}-1}{4}-\frac{T}{2}+\frac{\mathrm{e}^{2 T}-1}{2}=\frac{3\left(\mathrm{e}^{2 T}-1\right)}{4}-\frac{T}{2},
$$

we have the uniform convexity of $u(\cdot) \mapsto J(t, x ; u(\cdot))$. Therefore, optimal control unique exists and the value function is twice differentiable. In this case the optimal control $u^{*}(\cdot)$ admits a state feedback representation:

$$
u^{*}(s)=-\rho^{-1} P\left(s, X^{*}(s)\right), \quad s \in[t, T],
$$

with $P(\cdot, \cdot)$ solves the following quasi-Riccati equation:

$$
\left\{\begin{array}{l}
P_{t}(t, x)+x P_{x}(t, x)+P(t, x)+\sin 2 x-\rho^{-1} P_{x}(t, x) P(t, x)=0, \quad t \in[0, T], \\
P(T, x)=-\sin 2 x, \quad x \in \mathbb{R},
\end{array}\right.
$$

and $X^{*}(\cdot)$ is the solution to the following closed-loop system:

$$
\left\{\begin{array}{l}
\dot{X}^{*}(s)=X^{*}(s)-\rho^{-1} P\left(s, X^{*}(s)\right), \quad s \in[t, T] \\
X^{*}(t)=x
\end{array}\right.
$$


Example 7.3. Consider a one-dimensional controlled affine system

$$
\left\{\begin{array}{l}
\dot{X}(s)=\sin X(s)+u(s), \quad s \in[t, T] \\
X(t)=x
\end{array}\right.
$$

with cost functional

$$
J(t, x ; u(\cdot))=\int_{t}^{T}\left(\sqrt{1+|X(s)|^{2}}+\frac{R(s)}{2}|u(s)|^{2}\right) \mathrm{d} s .
$$

In this case, we have

$$
\left\{\begin{array}{l}
\dot{Y}(s)=Y(s) \cos X(s)-\frac{X(s)}{\sqrt{1+|X(s)|^{2}}} \\
Y(T)=0
\end{array}\right.
$$

Thus, we may take

$$
L_{A}=L_{Q}=1, \quad L_{G}=0 .
$$

Then

$$
L_{Y}=\mathrm{e}^{T}-1
$$

In the current case, by taking $\bar{G}=0$, we have

$$
G_{x x}(x)+\bar{G}=0 .
$$

Also, by letting $\bar{Q}(s)=\mathrm{e}^{T}-1$, we have

$$
Q_{x x}(s, x)+\bar{Q}(s)-L_{Y}\left|A_{x x}(s, x)\right|=\frac{1}{\left(1+x^{2}\right)^{\frac{3}{2}}}+\mathrm{e}^{T}-1-\left(\mathrm{e}^{T}-1\right)|\cos x| \geq 0 .
$$

Then

$$
\widehat{G}(t)=0,
$$

and

$$
\begin{aligned}
\widehat{Q}(s, t) & =\int_{s}^{T}\left[\int_{t}^{\tau} \int_{t}^{\tau}\left|\Phi_{\mathbf{A}}(T, r)\left(\mathrm{e}^{T}-1\right) \Phi_{\mathbf{A}}(T, r)\right|^{2} \mathrm{~d} r^{\prime} \mathrm{d} r\right]^{\frac{1}{2}} \mathrm{~d} \tau \\
& \leq\left(\mathrm{e}^{T}-1\right) \int_{s}^{T}\left[\int_{t}^{\tau} \int_{t}^{\tau} \mathrm{e}^{2(T-r)} \mathrm{e}^{2\left(T-r^{\prime}\right)} \mathrm{d} r^{\prime} \mathrm{d} r\right]^{\frac{1}{2}} \mathrm{~d} \tau \\
& =\left(\mathrm{e}^{T}-1\right) \int_{s}^{T} \frac{1}{2}\left[\mathrm{e}^{2(T-t)}-\mathrm{e}^{2(T-\tau)}\right] \mathrm{d} \tau \leq \frac{\mathrm{e}^{T}-1}{2} \mathrm{e}^{2 T}(T-s) .
\end{aligned}
$$

Hence, provided

$$
R(s)-[\widehat{G}(t)+\widehat{Q}(s)]=R(s)-\frac{\mathrm{e}^{T}-1}{2} \mathrm{e}^{2 T}(T-s) \geq \delta I, \quad 0 \leq s \leq T,
$$

we have the uniform convexity of $u(\cdot) \mapsto J(t, x ; u(\cdot))$. In the current case, the quasi-Riccati equation reads:

$$
\left\{\begin{array}{l}
P_{t}(t, x)+(\sin x) P_{x}(t, x)+(\cos x) P(t, x)+\sqrt{1+x^{2}}-P(t, x) P_{x}(t, x)=0, \quad(t, x) \in[0, T] \times \mathbb{R}^{n}, \\
P(T, x)=0, \quad x \in \mathbb{R}^{n} .
\end{array}\right.
$$

According to our result, the above quasi-Riccati equation admits a solution, provided

$$
R(s) \geq \frac{\mathrm{e}^{T}-1}{2} \mathrm{e}^{2 T}(T-s)+\delta I, \quad 0 \leq s \leq T .
$$


In this case, the optimal control admits a state feedback representation of form

$$
u^{*}(s)=-R(s)^{-1} P\left(s, X^{*}(s)\right), \quad s \in[t, T],
$$

with $X^{*}(\cdot)$ being the solution to the closed-loop system:

$$
\left\{\begin{array}{l}
\dot{X}^{*}(s)=\sin X^{*}(s)-R(s)^{-1} P\left(s, X^{*}(s)\right), \quad s \in[t, T], \\
X^{*}(t)=x,
\end{array}\right.
$$

\section{Concluding REMARKS}

We have presented some primitive results concerning what we call the affine-quadratic optimal control problems, which are a natural generalization of classical LQ problems, and also contains linear-convex problems as well as linear-semi-convex problems. Our results for linear state equation cover and substantially extend the known results for LQ problems and linear-convex problems in the literature. Further, we have obtained some results for the problems with (nonlinear) affine state equations. However, we point out that our results are far from satisfactory and there are many challenging problems left open. The most interesting one is the following: Is there an equivalence between the (unique) solvability of the optimality system, a two-point boundary value problem, and the corresponding quasi-Riccati equation? We hope to report some further relevant results in our future publications.

Acknowledgements. The authors would like to thank the two anonymous referees for their critical comments on the old version of the paper, which leads to the current much better version.

\section{REFERENCES}

[1] S.P. Banks and T. Cimen, Global optimal feedback control for general nonlinear systems with nonquadratic performance criteria. System Control Lett. 53 (2004) 327-346.

[2] S.P. Banks and T. Cimen, Optimal control of nonlinear systems, Optimization and Control with Applications. In vol. 96 of Appl. Optim. Springer, New York (2005) 353-367.

[3] H.T. Banks, B.M. Lewis and H.T. Tran, Nonlinear feedback controllers and compensators: a state-dependent Riccati equation approach. Comput. Optim. Appl. 37 (2007) 177-218.

[4] M. Bardi and I. Capuzzo-Dolcetta, Optimal Control and Viscosity Solutions of Hamilton-Jacobi-Bellman Equations. Birkhäuser, Boston (1997).

[5] M. Bardi and F. Da Lio, On the Bellman equation for some unbounded control problems. Nonlinear Differ. Eqs. Appl. 4 (1997) 491-510.

[6] L.M. Benveniste and J.A. Scheinkman, On the differentiability of the value function in dynamic models of economics. Econometrica 47 (1979) 727-732.

[7] L.D. Berkovitz, Optimal Control Theory. Springer-Verlag, New York (1974).

[8] J.F. Bonnans and A. Shapiro, Perturbation Analysis of Optimization Problems. Springer, New York (2000).

[9] P. Cannarsa and H. Frankowska, Some characterizatins of optimal trajecotries in control theory. SIAM J. Control Optim. 29 (1991) 1322-1347.

[10] T. Cimen, State-dependent Riccati equation (SDRE) control: a survey. Proc. 17th World Congress IFAC (2008) 3761-3775.

[11] H. Frankowska, Value Function in Optimal Control, Mathematical Control Theory, Part 1, 2 (2001) 516-653.

[12] T. Hildebrandt and L. Graves, Implicit functions and their differentials in general analysis. Trans. Amer. Math. Soc. 29 (1927) 127-153.

[13] Y. Hu and S. Peng, Solution of forward-backward stochastic differential equations. Probab. Theory Rel. Fields 103 (1995) 273-283.

[14] R.E. Kalman, Contributions to the theory of optimal control. Bol. Soc. Mat. Mexicana 5 (1960) 102-119.

[15] J. Ma and J. Yong, Forward-Backward Stochastic Differential Equations and Their Applications. Vol. 1702 of Lect. Notes Math. Springer-Verlag (1999).

[16] H. Qiu and J. Yong, Hamilton-Jacobi equations and two-person zero-sum differential games with unbounded controls. ESAIM: COCV 19 (2013) 404-437.

[17] J.P. Rincón-Zapatero and M.S. Santos, Differentiability of the value function in continuous-time economic models. J. Math. Anal. Appl. 394 (2012) 305-323. 
[18] J. Yong, Finding adapted solutions of forward-backward stochastic differential equations - method of continuation, Probab. Theory Rel. Fields 107 (1997) 537-572.

[19] J. Yong, Stochastic optimal control and forward-backward stochastic differential equations. Comput. Appl. Math. 21 (2002) 369-403.

[20] J. Yong, Forward backward stochastic differential equations with mixed initial and terminal conditions. Trans. AMS $\mathbf{3 6 2}$ (2010) 1047-1096.

[21] J. Yong and X.Y. Zhou, Stochastic Control: Hamiltonian Systems and HJB Equations. Springer-Verlag (1999).

[22] Y. You, A nonquadratic Bolza problem and a quasi-Riccati equation for distributed parameter systems. SIAM J. Control Optim. 25 (1987) 905-920.

[23] Y. You, Synthesis of time-variant optimal control with nonquadratic criteria. J. Math. Anal. Appl. 209 (1997) $662-682$.

[24] E. Zeidler, Nonlinear Functional Analysis and Its Applications, I: Fixed-Point Theorems. Springer-Verlag, New York (1986) 150-151.

[25] E. Zeidler, Nonlinear Functional Analysis and Its Applications, II/B: Nonlinear Monotone Operators. Springer-Verlag, New York (1990). 